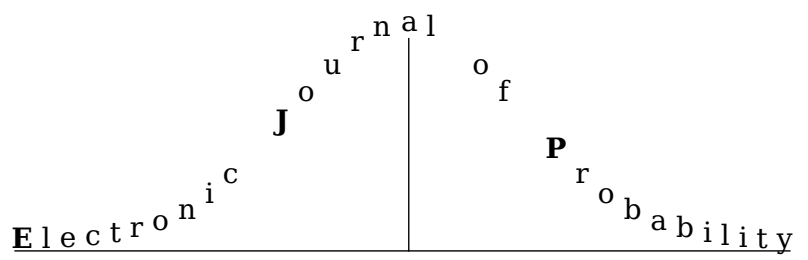

Electron. J. Probab. 26 (2021), article no. 91, 1-24.

ISSN: 1083-6489 https://doi.org/10.1214/21-EJP655

\title{
Reflected BSDEs with two optional barriers and monotone coefficient on general filtered space*
}

\author{
Tomasz Klimsiak $^{\dagger \ddagger} \quad$ Maurycy Rzymowski ${ }^{\ddagger}$
}

\begin{abstract}
We consider reflected backward stochastic differential equations with two optional barriers of class (D) satisfying Mokobodzki's separation condition, and coefficient which is only continuous and non-increasing. We assume that data are merely integrable and the terminal time is an arbitrary (possibly infinite) stopping time. We study the problem of the existence and uniqueness of solutions to the mentioned equations, and their connections with the value process in nonlinear Dynkin games.
\end{abstract}

Keywords: reflected backward stochastic differential equation; optional barriers; processes with regulated trajectories; Dynkin games; nonlinear expectation.

MSC2020 subject classifications: Primary 60H10, Secondary $60 \mathrm{G} 40$.

Submitted to EJP on January 19, 2020, final version accepted on June 7, 2021.

\section{Introduction}

Let $\mathbb{F}=\left(\mathcal{F}_{t}\right)_{t \geq 0}$ be a filtration satisfying the usual conditions and $T$ (terminal time) be an arbitrary (possibly infinite) $\mathbb{F}$-stopping time. We also consider as given an $\mathcal{F}_{T^{-}}$ measurable random variable $\xi$ (terminal condition), a real function $f$ (coefficient) defined on $\Omega \times \mathbb{R}^{+} \times \mathbb{R}$, which is $\mathbb{F}$-progressively measurable with respect to the first two variables, and $\mathbb{F}$-optional processes $L, U$ (barriers) of class (D) satisfying some separation condition. In the present paper, we consider reflected backward stochastic differential equations (RBSDE for short) which informally can be written in the form

$$
\left\{\begin{array}{l}
d Y_{t}=-f\left(t, Y_{t}\right) d t-d R_{t}+d M_{t} \text { on }[0, T], \quad \lim _{a \rightarrow \infty} Y_{T \wedge a}=\xi, \\
(Y, M, R) \in \mathcal{O} \times \mathcal{M}_{l o c} \times \mathcal{V}_{p}, \\
L \leq Y \leq U \text { on }[0, T], \quad d R \text { is "minimal". }
\end{array}\right.
$$

In (1.1), $\mathcal{O}$ (resp. $\mathcal{M}_{l o c}, \mathcal{V}_{p}$ ) is the space of $\mathbb{F}$-optional processes (resp. local $\mathbb{F}$-martingales, finite variation $\mathbb{F}$-predictable processes). We study the problem of the existence and

\footnotetext{
*This work was supported by Polish National Science Centre (Grant No. 2016/23/B/ST1/01543).

${ }^{\dagger}$ Institute of Mathematics, Polish Academy of Sciences, Poland.

${ }^{\ddagger}$ Faculty of Mathematics and Computer Science, Nicolaus Copernicus University, Poland.

E-mail: tomas@mat.umk.pl, maurycyrzymowski@mat.umk.pl
} 
uniqueness of solutions to (1.1) - also in case of one barrier, i.e. $U \equiv \infty-$ and their connections with nonlinear optimal stopping problem and nonlinear Dynkin games.

The notion of nonlinear RBSDEs was introduced by El Karoui et al. in [12] (one reflecting barrier) and by Cvitanic and Karatzas in [7] (two reflecting barriers). In both pioneering papers the authors considered bounded terminal time $T$, continuous barriers $L, U$ and filtration $\mathbb{F}$ generated by a given Brownian motion. In that case (1.1) may be formulated rigorously as follows:

$$
\left\{\begin{array}{l}
Y_{t}=\xi+\int_{t}^{T} f\left(r, Y_{r}, Z_{r}\right) d r+\int_{t}^{T} d R_{r}-\int_{t}^{T} Z_{r} d B_{r}, \quad t \in[0, T] \\
(Y, Z, R) \in \mathcal{O}_{c} \times \mathcal{O} \times \mathcal{V}_{c}, \\
L_{t} \leq Y_{t} \leq U_{t}, t \in[0, T], \quad \int_{0}^{T}\left(Y_{r}-L_{r}\right) d R_{r}^{+}=\int_{0}^{T}\left(U_{r}-Y_{r}\right) d R_{r}^{-}=0
\end{array}\right.
$$

where $\mathcal{O}_{c}$ (resp. $\mathcal{V}_{c}$ ) is the subspace of $\mathcal{O}$ (resp. $\mathcal{V}_{p}$ ) consisting of continuous processes. In $[7,12]$ it is assumed that the generator $f$ is Lipschitz continuous with respect to $y, z$ and the data are in $L^{2}$, that is $\xi, f(\cdot, 0,0)$, $\sup _{t<T}\left|L_{t}\right|$, and $\sup _{t<T}\left|U_{t}\right|$ (in the case of two barriers), are square-integrable. Note that in the special case of Brownian filtration each local $\mathbb{F}$-martingale $M$ is of the form $M=Z d B$, which allows one to consider $f$ depending also on $Z$.

In [7] an existence and uniqueness result for (1.2) is proved. Moreover, it is shown there that the solution is linked with Dynkin games via the formula

$$
Y_{\alpha}=\underset{\sigma \geq \alpha}{\operatorname{ess} \sup } \underset{\tau \geq \operatorname{sinf}}{\operatorname{ess}} E\left(\int_{\alpha}^{\tau \wedge \sigma} f\left(r, Y_{r}, Z_{r}\right) d r+L_{\sigma} \mathbf{1}_{\sigma<\tau}+U_{\tau} \mathbf{1}_{\tau \leq \sigma<T}+\xi \mathbf{1}_{\sigma=\tau=T} \mid \mathcal{F}_{\alpha}\right)
$$

holding for any stopping time $\alpha \in[0, T]$. When $U \equiv \infty$, the above formula reduces to the optimal stopping problem which was proved to be related to RBSDEs with one reflecting barrier in [12].

The theory of RBSDEs has been significantly developed over the last two decades and assumptions from the papers by Cvitanic and Karatzas [7] and by El Karoui et al. [12] were successively weakened. We shall provide a brief overview of the literature to show the main directions of relaxing of the standard assumptions considered in the pioneering papers. The case of càdlàg barriers is considered in [17, 22, 29]. RBSDEs with monotone generator satisfying weak growth condition are studied in $[22,28,34]$ and we refer the reader to $[5,21,22,34]$ for equations with $L^{p}$-data for $p \in[1,2]$. Equations with Brownian-Poisson filtration and càdlàg barriers were studied in $[16,18,19,20,36]$. The case of general, right-continuous filtration $\mathbb{F}$, monotone generator $f$ and $L^{1}$-data was studied in [23]. Equations with $T$ being an arbitrary stopping time were studied in [35] (Brownian filtration), in [1] (Brownian-Poisson filtration) and [24] (general filtration).

In most of the existing papers on RBSDEs càdlàg barriers are considered, and there are only few papers dealing with non-càdlàg case. Such equations with $L^{2}$-data and Lipschitz continuous generator were studied in [30] (Brownian filtration), in [13, 14, 31] (Brownian-Poisson filtration) and $[3,4,15]$ (general filtration). RBSDEs with $L^{1}$-data and optional barriers were considered only in $[25,26]$ in case of Brownian filtration and bounded terminal time.

As already mentioned, in the present paper we study the existence and uniqueness of solutions of class (D) to RBSDEs (1.1) with general filtration $\mathbb{F}$ and possibly infinite terminal time $T$. We also assume that $L$ and $U$ are $\mathbb{F}$-optional processes of class (D) satisfying Mokobodzki's condition - existence of a special semimartingale between the barriers - and such that

$$
\limsup _{a \rightarrow \infty} L_{T \wedge a} \leq \xi \leq \liminf _{a \rightarrow \infty} U_{T \wedge a} .
$$

As for $f$ and $V$, we will assume that they satisfy the following conditions. 
(H1) $E\left(\int_{0}^{T} d|V|_{r}+\int_{0}^{T}|f(r, 0)| d r\right)<\infty$,

(H2) for a.e. $t \in[0, T]$ the function $y \mapsto f(t, y)$ is non-increasing,

(H3) $y \mapsto f(t, y)$ is continuous for every $t \in[0, T]$,

(H4) $\int_{0}^{T}|f(r, y)| d r<\infty$ for all $y \in \mathbb{R}$.

One of the main novelty of the paper is that we study RBSDEs in the general framework described above. Some of the above conditions on the data were considered in the literature before in the context of RBSDEs, but here it is the first time that all these conditions are taken into account as one framework. Such far reaching generalization, as compared to the previous papers on RBSDEs, forces us to provide new proof techniques, and this is our second main contribution in the paper. Now, we shall describe briefly the new difficulties which appear in the paper and compare our methods with the already existing literature.

When dealing with RBSDEs with optional barriers, one of the main difficulty is the fact that the penalty method - one of the two known in the literature approaches to the existence problem for RBSDEs - is not applicable. Thus we are left with the second method, considered in [7, 12] and commonly used in case of optional barriers. This method consists of two steps: a) solving linear RBSDE by using the Snell envelope theory (in case of one barrier) or by using the Snell envelope theory combined with a decoupling method (in case of two barriers), b) applying a fixed point theorem (Banach contraction principle). In our framework part a) presents no difficulties and its proof proceeds analogously to the case of more regular data (see e.g. [14, 26]). The problems begin when we proceed to the nonlinear case since we can not apply fixed point argument the reason is twofold: $T$ may be infinite and $f$ is assumed to be merely non-decreasing with respect to $y$. To overcome this difficulty, we employ a natural method, coming out from PDEs, which is based on the iteration of equation in (1.1) admitting the following generic form

$$
d Y_{t}^{n}=-f_{n}\left(t, Y_{t}^{n-1}\right) d t-d R^{n}+d M_{t}^{n}
$$

However, since we do not assume any growth condition on $f$ with respect to $y$, in general, it is not true for solutions of (1.1) that $E \int_{0}^{T}\left|f\left(r, Y_{r}\right)\right| d r<\infty$ or $E|R|_{T}<\infty$, and even if this is true, it is very hard to show uniform integrability of the sequence $\left(f_{n}\left(\cdot, Y^{n-1}\right)\right)_{n \geq 1}$. Thus, it is by no means clear how to pass to the limit in (1.5) and in the Dynkin game formula (1.3) for the value process $Y^{n}$. In fact, by the comments in the foregoing, in general (1.3) is not even well defined, and we shall use an alternative formulation of (1.3) based on the nonlinear expectation (cf. (1.6)). The method we propose in the present paper for proving an existence result for (1.1) is based on a suitable localization (by means of a sequence of stopping times) of a particular iteration of the form (1.5), in case of one barrier, and a certain iteration of nonlinear decoupling system in case of two barriers (cf. (1.8)). Note here that one has to be very careful when choosing the proper localization because somehow we have to control uniformly in $n$ behavior of $Y^{n}$ at $T$ (possible infinite).

Now, we shall describe the content of the paper in more details. In Section 3 , for $f$ satisfying (H1)-(H4) we introduce the notion of nonlinear $f$-expectation

$$
\mathcal{E}_{\alpha, \beta}^{f}: L^{1}\left(\Omega, \mathcal{F}_{\beta}, P\right) \rightarrow L^{1}\left(\Omega, \mathcal{F}_{\alpha}, P\right)
$$

associated with BSDE (1.1) with no reflection, and we prove its basic properties. Here $\alpha \leq \beta$ are stopping times. 
In Section 4, we give a definition of a solution to (1.1). We are looking for a triple $(Y, M, R) \in \mathcal{O} \times \mathcal{M}_{l o c} \times \mathcal{V}_{p}$ such that $Y$ is a regulated (làdlàg) process such that

$$
Y_{t}=Y_{T \wedge a}+\int_{t}^{T \wedge a} f\left(r, Y_{r}\right) d r+\int_{t}^{T \wedge a} d R_{r}-\int_{t}^{T \wedge a} d M_{r}, \quad t \in[0, T \wedge a]
$$

for every $a \geq 0$, and moreover, $Y$ satisfies the terminal condition of the form

$$
Y_{T \wedge a} \rightarrow \xi, \quad a \rightarrow \infty .
$$

We also require that $R$ is minimal in the sense that for every $a \geq 0$,

$$
\begin{aligned}
\int_{0}^{T \wedge a} & \left(Y_{r-}-\vec{L}_{r}\right) d R_{r}^{*,+}+\sum_{0 \leq r<T \wedge a}\left(Y_{r}-L_{r}\right) \Delta^{+} R_{r}^{+} \\
& +\int_{0}^{T \wedge a}\left(\underline{U}_{r}-Y_{r-}\right) d R_{r}^{*,-}+\sum_{0 \leq r<T \wedge a}\left(U_{r}-Y_{r}\right) \Delta^{+} R_{r}^{-}=0,
\end{aligned}
$$

where $R=R^{+}-R^{-}$is the Jordan decomposition of $R, \vec{L}_{r}=\lim \sup _{s \uparrow r} X_{s}, \underline{U}_{r}=$ $\liminf \operatorname{in}_{s \uparrow} X_{s}$, and $R^{*,+}$ (resp. $R^{*,-}$ ) is the càdlàg part of the process $R^{+}$(resp. $R^{-}$). We prove that there exists at most one solution $(Y, M, R)$ to $\operatorname{RBSDE}(1.1)$ such that $Y$ is of class (D).

In Section 5, we prove that under (H1)-(H4) and $E|\xi|<\infty$, there exists a solution $(Y, M, R)$ to RBSDE (1.1) with one reflecting lower barrier $L$ such that $Y$ is of class (D). In this case $R$ is an increasing process. We also show that for every stopping time $\alpha \leq T$,

$$
Y_{\alpha}=\underset{\tau \geq \alpha}{\operatorname{ess} \sup } \mathcal{E}_{\alpha, \tau}^{f}\left(L_{\tau} \mathbf{1}_{\tau<T}+\xi \mathbf{1}_{\tau=T}\right) .
$$

Let us stress here that in general $E R_{T}$ and $E \int_{0}^{T}\left|f\left(r, Y_{r}\right)\right| d r$ are infinite. We give necessary and sufficient condition for which these integrals are finite. We show that if this is the case, then

$$
Y_{\alpha}=\underset{\tau \geq \alpha}{\operatorname{ess} \sup } E\left(\int_{\alpha}^{\tau} f\left(r, Y_{r}\right) d r+L_{\tau} \mathbf{1}_{\tau<T}+\xi \mathbf{1}_{\tau=T} \mid \mathcal{F}_{\alpha}\right) .
$$

In Section 6, we are focused on our main goal, i.e. problem (1.1) (with two reflecting barriers). We first show that each solution to (1.1) such that $Y$ is of class (D) admits the representation

$$
Y_{\alpha}=\operatorname{ess~sup}_{\rho=(\tau, H) \in \mathcal{S}_{\alpha}} \operatorname{essinf}_{\delta=(\sigma, G) \in \mathcal{S}_{\alpha}} \mathcal{E}_{\alpha, \tau \wedge \sigma}^{f}\left(L_{\rho}^{u} \mathbf{1}_{\{\tau \leq \sigma<T\}}+U_{\delta}^{l} \mathbf{1}_{\{\sigma<\tau\}}+\xi \mathbf{1}_{\{\tau=\sigma=T\}}\right),
$$

where $\rho, \delta$ are the so called stopping systems (see Section 6), and

$$
L_{\rho}^{u}=L_{\tau} \mathbf{1}_{H}+\overleftarrow{L}_{\tau} \mathbf{1}_{H^{c}}, \quad U_{\rho}^{l}=U_{\tau} \mathbf{1}_{G}+U_{\tau} \mathbf{1}_{G^{c}}
$$

Using this representation, we prove a stability result for (1.1). To prove the existence of a solution, we consider the nonlinear decoupling system

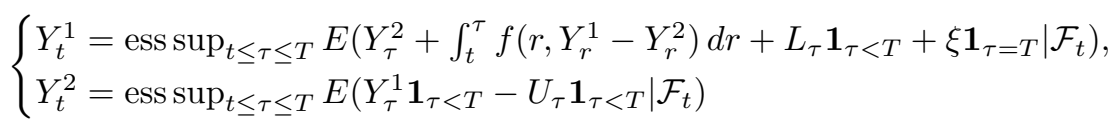

introduced in the linear case ( $f \equiv 0$ ) by Bismut [6]. Since, as we mentioned before, the integral in (1.7) may be infinite, we reformulate (1.7) as the following system of RBSDEs with one reflecting lower barrier:

$$
\left\{\begin{array}{l}
d Y_{t}^{1}=-f\left(t, Y_{t}^{1}-Y_{t}^{2}\right) d r-d R_{t}^{1}+d M_{t}^{1} \text { on }[0, T], \quad Y_{T}^{1}=\xi \\
d Y_{t}^{2}=-d R_{t}^{2}+d M_{t}^{2} \text { on }[0, T], \quad Y_{T}^{2}=0 \\
Y^{2}+L \leq Y^{1}, \quad Y^{1}-U \leq Y^{2}
\end{array}\right.
$$


Using the results of Section 5, we prove that under (H1)-(H4), (1.4) and Mokobodzki's condition (the existence of a special semimartingale between the barriers) there exists a solution $\left(Y^{1}, M^{1}, R^{1}\right),\left(Y^{2}, M^{2}, R^{2}\right)$ to (1.8) such that $Y^{1}, Y^{2}$ are of class (D). Next we show that the triple

$$
(Y, M, R):=\left(Y^{1}, M^{1}, R^{1}\right)-\left(Y^{2}, M^{2}, R^{2}\right)
$$

is a solution to (1.1). We also give a necessary and sufficient condition under which $E|R|_{T}$ and $E \int_{0}^{T}\left|f\left(r, Y_{r}\right)\right| d r$ are finite. Finally, using our existence and stability result, we show that there exists a solution $(Y, M, R)$ to (1.1) such that $Y$ is of class (D) even if $f, V$ do not satisfy (H1). It is worth mentioning here that in general, without (H1) there is no solution to equation of type (1.1) with no reflection. In other words, we show that for the existence of solutions to reflected BSDEs weaker assumptions on $f$ are needed than for the existence of solutions to related BSDEs.

\section{Notation and standing assumptions}

Let $a \geq 0$. We say that a function $y:[0, a] \rightarrow \mathbb{R}$ is regulated if the limit $y_{t+}=\lim _{u \downarrow t} y_{u}$ exists for every $t \in[0, a)$, and the limit $y_{s-}=\lim _{u \uparrow s} y_{u}$ exists for every $s \in(0, a]$. For any regulated function $y$ on $[0, a]$ we set $\Delta^{+} y_{t}=y_{t+}-y_{t}$ if $0 \leq t<a$, and $\Delta y_{s}=y_{s}-y_{s-}$ if $0<s \leq a$. It is known that each regulated function is bounded and has at most countably many discontinuities (see, e.g., [10, Chapter 2, Corollary 2.2]). For $x \in \mathbb{R}$, we set $\operatorname{sgn}(x)=\mathbf{1}_{x \neq 0} x /|x|$.

Let $(\Omega, \mathcal{F}, P)$ be a probability space, $\mathbb{F}=\left(\mathcal{F}_{t}\right)_{t \geq 0}$ be a filtration satisfying the usual conditions and let $T$ be an $\mathbb{F}$-stopping time. For $a \geq 0$, we set $T_{a}=T \wedge a$. For fixed stopping times $\sigma, \tau$ we denote by $\mathcal{T}_{\sigma, \tau}$ the set of all $\mathbb{F}$-stopping times taking values in $[\sigma, \tau]$. We also set $\mathcal{T}_{\tau}=\mathcal{T}_{\tau, T}, \mathcal{T}^{\tau}=\mathcal{T}_{0, \tau}$, and $\mathcal{T}=\mathcal{T}^{T}$. For given $\beta \in \mathcal{T}$, we let $L^{1}\left(\Omega, \mathcal{F}_{\beta}, P\right)$ denote a space of $\mathcal{F}_{\beta}$ measurable random variables $\xi$ satisfying $E|\xi|<\infty$. An increasing sequence $\left\{\tau_{k}\right\} \subset \mathcal{T}$ is called a chain (on $[0, T]$ ) if

$$
\forall \omega \in \Omega \quad \exists n \in \mathbb{N} \quad \forall k \geq n \quad \tau_{k}(\omega)=T .
$$

We say that an $\mathbb{F}$-progressively measurable process $X$ is of class (D) (on $[0, T]$ ) if the family $\left\{X_{\tau}, \tau \in \mathcal{T}, \tau<\infty\right\}$ is uniformly integrable. We equip the space of processes of class (D) with the norm $\|X\|_{1}=\sup _{\tau \in \mathcal{T}, \tau<\infty} E\left|X_{\tau}\right|$. In the sequel, in case $X_{\infty}$ is not defined, we set $X_{\infty}=0$.

We denote by $\mathcal{M}$ (resp. $\mathcal{M}_{\text {loc }}$ ) the set of all $\mathbb{F}$ - martingales (resp. local martingales) such that $M_{0}=0$, and by $\mathcal{V}\left(\right.$ resp. $\mathcal{V}^{+}$) the space of all $\mathbb{F}$ - progressively measurable processes of finite variation (resp. increasing) such that $V_{0}=0 . \mathcal{V}^{1}$ (resp. $\mathcal{V}^{1,+}$ ) is the set of processes $V \in \mathcal{V}$ (resp. $V \in \mathcal{V}^{+}$) such that $E|V|_{T}<\infty$, where $|V|_{T}$ stands for the total variation of $V$ on $[0, T] . \mathcal{V}_{p}$ (resp. $\mathcal{V}_{p}^{+}$) is the space of all predictable $V \in \mathcal{V}$ (resp. $V \in \mathcal{V}^{+}$). For $V \in \mathcal{V}$, by $V^{*}$ we denote the càdlàg part of the process $V$, and by $V^{d}$ its purely jumping part consisting of right jumps, i.e.

$$
V_{t}^{d}=\sum_{s<t} \Delta^{+} V_{s}, \quad V_{t}^{*}=V_{t}-V_{t}^{d}, \quad t \in[0, T]
$$

In the whole paper all relations between random variables are understood to hold $P$-a.s. For processes $X$ and $Y$, we write $X \leq Y$ if $X_{t} \leq Y_{t}, t \in\left[0, T_{a}\right], a \geq 0$. For a process $X$, we set $\vec{X}_{s}=\lim \sup _{r \uparrow s} X_{r}, \overleftarrow{X}_{s}=\operatorname{lim\operatorname {sup}_{r\downarrow s}} X_{r}, \underline{X}_{s}=\liminf _{r \uparrow s} X_{r}$ and $\underline{X}_{s}=\liminf _{r \downarrow s} X_{r}, s \in\left[0, T_{a}\right], a \geq 0$. By [8, Theorem 90, page 143], if $X$ is an optional process of class (D), then $\overleftarrow{X}, X$ are progressively measurable, and $\vec{X}, X$ are predictable processes. 
Throughout the paper, $L$ and $U$ are $\mathbb{F}$-adapted optional processes of class (D), and $\xi$ is $\mathcal{F}_{T}$-measurable random variable. We always assume (except Section 5) that (1.4) is satisfied which implies that $E|\xi|<\infty$. The generator (coefficient, driver) is a map

$$
\Omega \times[0, T] \times \mathbb{R} \ni(\omega, t, y) \mapsto f(\omega, t, y) \in \mathbb{R},
$$

which is $\mathbb{F}$-progressively measurable for fixed $y$. For an $\mathbb{F}$-adapted regulated process $Y$, we set

$$
f_{Y}(t)=f\left(t, Y_{t}\right), \quad t \in[0, T]
$$

As for $V$, we always assume that $V \in \mathcal{V}$.

\section{BSDEs and nonlinear expectation}

Definition 3.1. We say that a pair $(Y, M)$ of $\mathbb{F}$-adapted processes is a solution of the backward stochastic differential equation on the interval $[0, T]$ with right-hand side $f+d V$ and terminal value $\xi\left(B S D E^{T}(\xi, f+d V)\right.$ for short) if

(a) $Y$ is regulated and $M \in \mathcal{M}_{\text {loc }}$,

(b) $\int_{0}^{T_{a}}\left|f\left(r, Y_{r}\right)\right| d r<+\infty$ for any $a \geq 0$,

(c) for every $a \geq 0$,

$$
Y_{t}=Y_{T_{a}}+\int_{t}^{T_{a}} f\left(r, Y_{r}\right) d r+\int_{t}^{T_{a}} d V_{r}-\int_{t}^{T_{a}} d M_{r}, \quad t \in\left[0, T_{a}\right],
$$

(d) $\lim _{a \rightarrow \infty} Y_{T_{a}}=\xi$ a.s.

Remark 3.2. Existence, uniqueness and some other properties of solutions to equation $\operatorname{BSDE}^{T}(\xi, f+d V)$ we will use later on follow from [24] (see also [27]). In these papers it is assumed that $V$ is càdlàg but the results of [24, 27] may be applied to the case when $V$ is regulated by a simple change of variables. Indeed, if $(\bar{Y}, \bar{M})$ is a solution to $\operatorname{BSDE}^{T}\left(\xi, f^{*}+d V^{*}\right)$ with

$$
f^{*}(t, y)=f\left(t, y+V_{t}^{d}\right)
$$

then $(Y, \bar{M})$ with $Y=\bar{Y}+V^{d}$ is a solution to $\operatorname{BSDE}^{T}(\xi, f+d V)$.

We now introduce the notion of nonlinear expectation in our general framework.

Definition 3.3 (Nonlinear expectation). Assume (H1)-(H4). Let $\alpha, \beta \in \mathcal{T}, \alpha \leq \beta$. We say that an operator

$$
\mathcal{E}_{\alpha, \beta}^{f}: L^{1}\left(\Omega, \mathcal{F}_{\beta}, P\right) \rightarrow L^{1}\left(\Omega, \mathcal{F}_{\alpha}, P\right)
$$

is a nonlinear $f$-expectation if for any $\xi \in L^{1}\left(\Omega, \mathcal{F}_{\beta}, P\right)$, we have $\mathcal{E}_{\alpha, \beta}^{f}(\xi)=Y_{\alpha}$, where $(Y, M)$ is the unique solution of $\operatorname{BSDE}^{\beta}(\xi, f)$.

We say that a càdlàg process $X$ of class (D) is an $\mathcal{E}^{f}$-supermartingale (resp. $\mathcal{E}^{f}$ submartingale) on $[\alpha, \beta]$ if $\mathcal{E}_{\sigma, \tau}^{f}\left(X_{\tau}\right) \leq X_{\sigma}$ (resp. $\mathcal{E}_{\sigma, \tau}^{f}\left(X_{\tau}\right) \geq X_{\sigma}$ ) for all $\tau, \sigma \in \mathcal{T}$ such that $\alpha \leq \sigma \leq \tau \leq \beta$. $X$ is called an $\mathcal{E}^{f}$-martingale on $[\alpha, \beta]$ if it is both $\mathcal{E}^{f}$-supermartingale and $\mathcal{E}^{f}$-submartingale on $[\alpha, \beta]$.

For a given finite variation process $V$ and stopping times $\alpha, \beta(\alpha \leq \beta)$ we denote by $|V|_{\alpha, \beta}$ the total variation of the process $V$ on $[\alpha, \beta]$. By $\operatorname{BSDE}^{\alpha, \beta}(\xi, f+d V)$ we denote the problem (a)-(d) of Definition 3.1 but with $0, T$ replaced by $\alpha, \beta$, respectively.

In Proposition 3.4 we gather some properties of nonlinear $f$-expectation which will be needed later on. These properties are direct consequences of the existence, uniqueness, stability, and comparison theorems for BSDEs proved in [24]. Properties (i)-(iii) below were proved in [27, Proposition 5.6]. 
Proposition 3.4 (Basic properties of nonlinear expectation). Assume that $f$ satisfies (H1)-(H4) and $\alpha, \beta \in \mathcal{T}, \alpha \leq \beta$.

(i) Let $\xi \in L^{1}\left(\Omega, \mathcal{F}_{\beta}, P\right)$ and $V$ be an $\mathbb{F}$-adapted finite variation process such that $V_{\alpha}=0$ and $E|V|_{\alpha, \beta}<\infty$. Then there exists a unique solution $(X, N)$ of $\operatorname{BSDE}^{\alpha, \beta}(\xi, f+d V)$. Moreover, if $V$ (resp. $-V$ ) is an increasing process, then $X$ is an $\mathcal{E}^{f}$-supermartingale (resp. $\mathcal{E}^{f}$-submartingale) on $[\alpha, \beta]$.

(ii) If $\xi_{1}, \xi_{2} \in L^{1}\left(\Omega, \mathcal{F}_{\beta}, P\right)$ and $\xi_{1} \leq \xi_{2}$, then $\mathcal{E}_{\alpha, \beta}^{f}\left(\xi_{1}\right) \leq \mathcal{E}_{\alpha, \beta}^{f}\left(\xi_{2}\right)$.

(iii) If $f_{1}, f_{2}$ satisfy (H1)-(H4), $\alpha, \beta_{1}, \beta_{2} \in \mathcal{T}, \alpha \leq \beta_{1} \leq \beta_{2}, \xi_{1} \in L^{1}\left(\Omega, \mathcal{F}_{\beta_{1}}, P\right), \xi_{2} \in$ $L^{1}\left(\Omega, \mathcal{F}_{\beta_{2}}, P\right)$, then

$$
\begin{aligned}
\left|\mathcal{E}_{\alpha, \beta_{1}}^{f_{1}}\left(\xi_{1}\right)-\mathcal{E}_{\alpha, \beta_{2}}^{f_{2}}\left(\xi_{2}\right)\right| \leq & E\left(\left|\xi_{1}-\xi_{2}\right|+\int_{\alpha}^{\beta_{1}}\left|f^{1}\left(r, Y_{r}^{1}\right)-f^{2}\left(r, Y_{r}^{1}\right)\right| d r\right. \\
& \left.+\int_{\beta_{1}}^{\beta_{2}}\left|f^{2}\left(r, Y_{r}^{2}\right)\right| d r \mid \mathcal{F}_{\alpha}\right)
\end{aligned}
$$

where $Y_{t}^{1}=\mathcal{E}_{t \wedge \beta_{1}, \beta_{1}}^{f^{1}}\left(\xi_{1}\right), Y_{t}^{2}=\mathcal{E}_{t \wedge \beta_{2}, \beta_{2}}^{f^{2}}\left(\xi_{2}\right)$,

(iv) Let $\xi \in L^{1}\left(\Omega, \mathcal{F}_{\beta}, P\right)$. For every $A \in \mathcal{F}_{\alpha}$,

$$
\mathbf{1}_{A} \mathcal{E}_{\alpha, \beta}^{f}(\xi)=\mathcal{E}_{\alpha, \beta}^{f_{A}}\left(\mathbf{1}_{A} \xi\right),
$$

where $f_{A}(t, y)=f(t, y) \mathbf{1}_{A} \mathbf{1}_{t \geq \alpha}$,

(v) Let $\xi \in L^{1}\left(\Omega, \mathcal{F}_{\beta}, P\right)$. For every $\gamma \in \mathcal{T}$ such that $\gamma \geq \beta$,

$$
\mathcal{E}_{\alpha, \beta}^{f}(\xi)=\mathcal{E}_{\alpha, \gamma}^{f^{\beta}}(\xi)
$$

where $f^{\beta}(t, y)=f(t, y) \mathbf{1}_{t \leq \beta}$.

\section{Definition of a solution and a comparison result}

Definition 4.1 (BSDEs with one reflecting barrier). We say that a triple $(Y, M, K)$ of F-adapted processes is a solution of a reflected backward stochastic differential equation on the interval $[0, T]$ with right-hand side $f+d V$, terminal value $\xi$ and lower barrier $L$ $\left(\underline{R B S D E} E^{T}(\xi, f+d V, L)\right.$ for short) if

(a) $Y$ is regulated and $M \in \mathcal{M}_{\text {loc }}$,

(b) $K \in \mathcal{V}_{p}^{+}, L_{t} \leq Y_{t}, t \in\left[0, T_{a}\right], a \geq 0$, and

$$
\int_{0}^{T_{a}}\left(Y_{r-}-\vec{L}_{r}\right) d K_{r}^{*}+\sum_{0 \leq r<T_{a}}\left(Y_{r}-L_{r}\right) \Delta^{+} K_{r}=0, \quad a \geq 0
$$

(c) $\int_{0}^{T_{a}}\left|f\left(r, Y_{r}\right)\right| d r<+\infty$ for every $a \geq 0$,

(d) for any $a \geq 0$,

$$
Y_{t}=Y_{T_{a}}+\int_{t}^{T_{a}} f\left(r, Y_{r}\right) d r+\int_{t}^{T_{a}} d V_{r}+\int_{t}^{T_{a}} d K_{r}-\int_{t}^{T_{a}} d M_{r}, \quad t \in\left[0, T_{a}\right],
$$

(e) $\lim _{a \rightarrow \infty} Y_{T_{a}}=\xi$ a.s. 
Definition 4.2 (BSDEs with two reflecting barriers). We say that a triple $(Y, M, R)$ of F-adapted processes is a solution of a reflected backward stochastic differential equation on the interval $[0, T]$ with right-hand side $f+d V$, terminal value $\xi$, lower barrier $L$ and upper barrier $U\left(R B S D E^{T}(\xi, f+d V, L, U)\right.$ for short) if

(a) $Y$ is regulated and $M \in \mathcal{M}_{\text {loc }}$,

(b) $R \in \mathcal{V}_{p}, L_{t} \leq Y_{t} \leq U_{t}, t \in\left[0, T_{a}\right], a \geq 0$, and

$$
\begin{aligned}
\int_{0}^{T_{a}} & \left(Y_{r-}-\vec{L}_{r}\right) d R_{r}^{*,+}+\sum_{0 \leq r<T_{a}}\left(Y_{r}-L_{r}\right) \Delta^{+} R_{r}^{+} \\
& +\int_{0}^{T_{a}}\left(\underline{U}_{r}-Y_{r-}\right) d R_{r}^{*,-}+\sum_{0 \leq r<T_{a}}\left(U_{r}-Y_{r}\right) \Delta^{+} R_{r}^{-}=0, \quad a \geq 0
\end{aligned}
$$

where $R=R^{+}-R^{-}$is the Jordan decomposition of $R$,

(c) $\int_{0}^{T_{a}}\left|f\left(r, Y_{r}\right)\right| d r<+\infty$ for every $a \geq 0$,

(d) for any $a \geq 0$,

$$
Y_{t}=Y_{T_{a}}+\int_{t}^{T_{a}} f\left(r, Y_{r}\right) d r+\int_{t}^{T_{a}} d V_{r}+\int_{t}^{T_{a}} d R_{r}-\int_{t}^{T_{a}} d M_{r}, \quad t \in\left[0, T_{a}\right],
$$

(e) $\lim _{a \rightarrow \infty} Y_{T_{a}}=\xi$ a.s.

Proposition 4.3 (Comparison result). Let $\left(Y^{i}, M^{i}, R^{i}\right)$ be a solution of $\operatorname{RBSDE}^{T}\left(\xi^{i}, f^{i}+\right.$ $\left.d V^{i}, L^{i}, U^{i}\right), i=1,2$. Assume that $f^{1}$ satisfies (H2) and $\xi^{1} \leq \xi^{2}, f^{1}\left(\cdot, Y^{2}\right) \leq f^{2}\left(\cdot, Y^{2}\right)$ $d t \otimes d P$-a.s., $d V^{1} \leq d V^{2}, L^{1} \leq L^{2}, U^{1} \leq U^{2}$. If $\left(Y^{1}-Y^{2}\right)^{+}$is of class (D), then $Y^{1} \leq Y^{2}$.

Proof. By (H2) and the fact that $f^{1}\left(\cdot, Y^{2}\right) \leq f^{2}\left(\cdot, Y^{2}\right) d t \otimes d P$-a.s. we have

$$
\mathbf{1}_{\left\{Y_{r}^{1}>Y_{r}^{2}\right\}}\left(f^{1}\left(r, Y_{r}^{1}\right)-f^{2}\left(r, Y_{r}^{2}\right)\right) \leq \mathbf{1}_{\left\{Y_{r}^{1}>Y_{r}^{2}\right\}}\left(f^{1}\left(r, Y_{r}^{1}\right)-f^{1}\left(r, Y_{r}^{2}\right)\right) \leq 0 .
$$

By the minimality condition for $R^{1}, R^{2}$ and the assumption that $L^{1} \leq L^{2}$ and $U^{1} \leq U^{2}$,

$$
\mathbf{1}_{\left\{Y_{r-}^{1}>Y_{r-}^{2}\right\}} d\left(R_{r}^{1}-R_{r}^{2}\right)^{*} \leq \mathbf{1}_{\left\{Y_{r-}^{1}>Y_{r-}^{2}\right\}} d R^{1, *,+}+\mathbf{1}_{\left\{Y_{r-}^{1}>Y_{r-}^{2}\right\}} d R^{2,-, *}=0
$$

and

$$
\mathbf{1}_{\left\{Y_{r}^{1}>Y_{r}^{2}\right\}} \Delta^{+}\left(R_{r}^{1}-R_{r}^{2}\right) \leq \mathbf{1}_{\left\{Y_{r}^{1}>Y_{r}^{2}\right\}} \Delta^{+} R_{r}^{1,+}+\mathbf{1}_{\left\{Y_{r}^{1}>Y_{r}^{2}\right\}} \Delta^{+} R_{r}^{2,-}=0 .
$$

By [25, Corollary A.5], for all $a \geq 0$ and stopping times $\sigma, \tau \in \mathcal{T}^{T_{a}}$ such that $\sigma \leq \tau$ we have

$$
\begin{aligned}
\left(Y_{\sigma}^{1}-Y_{\sigma}^{2}\right)^{+} \leq & \left(Y_{\tau}^{1}-Y_{\tau}^{2}\right)^{+}+\int_{\sigma}^{\tau} \mathbf{1}_{\left\{Y_{r}^{1}>Y_{r}^{2}\right\}}\left(f^{1}\left(r, Y_{r}^{1}\right)-f^{2}\left(r, Y_{r}^{2}\right)\right) d r \\
& +\int_{\sigma}^{\tau} \mathbf{1}_{\left\{Y_{r-}^{1}>Y_{r-}^{2}\right\}} d\left(V_{r}^{1}-V_{r}^{2}\right)^{*}+\sum_{\sigma \leq r<\tau} \int_{\sigma}^{\tau} \mathbf{1}_{\left\{Y_{r}^{1}>Y_{r}^{2}\right\}} \Delta^{+}\left(V_{r}^{1}-V_{r}^{2}\right) \\
& +\int_{\sigma}^{\tau} \mathbf{1}_{\left\{Y_{r-}^{1}>Y_{r-}^{2}\right\}} d\left(R_{r}^{1}-R_{r}^{2}\right)^{*}+\sum_{\sigma \leq r<\tau} \int_{\sigma}^{\tau} \mathbf{1}_{\left\{Y_{r}^{1}>Y_{r}^{2}\right\}} \Delta^{+}\left(R_{r}^{1}-R_{r}^{2}\right) \\
& -\int_{\sigma}^{\tau} \mathbf{1}_{\left\{Y_{r-}^{1}>Y_{r-}^{2}\right\}} d\left(M_{r}^{1}-M_{r}^{2}\right) .
\end{aligned}
$$

By the above inequality, (4.1)-(4.3) and the assumption that $d V_{1} \leq d V_{2}$, we have

$$
\left(Y_{\sigma}^{1}-Y_{\sigma}^{2}\right)^{+} \leq\left(Y_{\tau}^{1}-Y_{\tau}^{2}\right)^{+}-\int_{\sigma}^{\tau} \mathbf{1}_{\left\{Y_{r-}^{1}>Y_{r-}^{2}\right\}} d\left(M_{r}^{1}-M_{r}^{2}\right)
$$


Let $\left\{\tau_{k}\right\}$ be a fundamental sequence, on $\left[0, T_{a}\right]$, for the local martingale $M^{1}-M^{2}$. By (4.4) with $\tau$ replaced by $\tau_{k} \geq \sigma$, we get

$$
\left(Y_{\sigma}^{1}-Y_{\sigma}^{2}\right)^{+} \leq\left(Y_{\tau_{k}}^{1}-Y_{\tau_{k}}^{2}\right)^{+}-\int_{\sigma}^{\tau_{k}} \mathbf{1}_{\left\{Y_{r-}^{1}>Y_{r-}^{2}\right\}} d\left(M_{r}^{1}-M_{r}^{2}\right), k \in \mathbb{N} .
$$

Taking the expectation and then letting $k \rightarrow \infty$ we obtain $E\left(Y_{\sigma}^{1}-Y_{\sigma}^{2}\right)^{+} \leq E\left(Y_{T_{a}}^{1}-Y_{T_{a}}^{2}\right)^{+}$ for $a \geq 0$. Letting $a \rightarrow \infty$ yields $E\left(Y_{\sigma}^{1}-Y_{\sigma}^{2}\right)^{+}=E\left(\xi^{1}-\xi^{2}\right)^{+}=0$. In both limits we used the fact that $\left(Y^{1}-Y^{2}\right)^{+}$is of class (D). Therefore, by the Section Theorem (see, e.g., [8, Chapter IV, Theorem 86]), $\left(Y_{t}^{1}-Y_{t}^{2}\right)^{+}=0, t \in[0, T]$. Hence $Y^{1} \leq Y^{2}$.

\section{Existence of a solution for BSDEs with one reflecting barrier}

In this section, we focus on the existence problem for nonlinear RBSDEs with one reflecting barrier. Therefore, exceptionally, in this chapter, we assume that, instead of (1.4), it holds

$$
\limsup _{a \rightarrow \infty} L_{T \wedge a} \leq \xi
$$

First, we prove that the process $Y$ defined by means of the Snell envelope is the first component of the solution to linear RBSDE. The proof is relatively standard and analogous to that in [15] with one exception that some extra effort is needed to prove behavior of $Y$ at $T$ (we allow infinity). After a priori estimates (Section 5.2) we proceed to the proof of an existence result for nonlinear RBSDEs. We divide the proof into three steps which we formulate as three separate results. In Proposition 5.3 we assume that $f$ is Lipschitz continuous in $y$ with Lipschitz constant depending on time. In Proposition 5.4, we assume that $f$ is merely bounded from below. Finally, in Theorem 5.5, which is the main result of this section, we consider the problem in full generality, i.e. under assumptions (H1)-(H4). We close the section by showing that the first component of the solution to nonlinear RBSDE is the value process in nonlinear optimal stopping problem.

\subsection{Existence results for linear equations}

Recall (see e.g. [9, page 417]) that if we set for any $\alpha \in \mathcal{T}$,

$$
Y(\alpha)=\underset{\tau \geq \alpha}{\operatorname{essipup}} E\left(L_{\tau} \mid \mathcal{F}_{\alpha}\right)
$$

then there exists a positive supermartingale $Y$ of class (D) on $[0, T]$ such that for every $\alpha \in \mathcal{T}, Y(\alpha)=Y_{\alpha}$. Moreover, for any $\alpha, \sigma \in \mathcal{T}$ such that $\alpha \leq \sigma$,

$$
Y_{\alpha}=\operatorname{ess~sup}_{\tau \in \mathcal{T}_{\alpha, \sigma}} E\left(L_{\tau} \mathbf{1}_{\tau<\sigma}+Y_{\sigma} \mathbf{1}_{\tau=\sigma} \mid \mathcal{F}_{\alpha}\right) .
$$

Proposition 5.1 (Linear equations and Snell envelope representation). Assume that $\xi \in L^{1}\left(\Omega, \mathcal{F}_{T}, P\right)$ and $V \in \mathcal{V}^{1}$. Then there exists a unique solution $(Y, M, K)$ of the problem $\underline{\operatorname{RBSDE}}^{T}(\xi, d V, L)$, such that $Y$ is of class (D). Moreover,

$$
Y_{\alpha}=\operatorname{esssup}_{\tau \in \mathcal{T}_{\alpha}} E\left(\int_{\alpha}^{\tau} d V_{r}+L_{\tau} \mathbf{1}_{\{\tau<T\}}+\xi \mathbf{1}_{\{\tau=T\}} \mid \mathcal{F}_{\alpha}\right), \quad \alpha \in \mathcal{T} .
$$

Proof. Observe that $(Y, M, K)$ is a solution to $\operatorname{RBSDE}^{T}(\xi, d V, L)$ if and only if $(Y+V, M, K)$ is a solution to $\underline{\operatorname{RBSDE}}^{T}\left(\xi+V_{T}, 0, L+V\right)$. Therefore, without loss of generality, we may assume that $V \equiv 0$. Defining $Y$ by the right-hand side of (5.3), and using (5.2) and the Mertens decomposition theorem (see [32]), we get that there exist $K \in \mathcal{V}_{p}^{+}, M \in \mathcal{M}_{\text {loc }}$ such that for any $a>0$,

$$
Y_{t}=Y_{T_{a}}+\int_{t}^{T_{a}} d K_{r}-\int_{t}^{T_{a}} d M_{r}, \quad t \in\left[0, T_{a}\right]
$$


Clearly, conditions (a) and (c) of Definition 4.1 are satisfied. Now, we shall prove that conditions (b) and (d) of Definition 4.1 are satisfied. Once this is done the existence part of the proof is complete. Applying [15, Lemma 3.2, Lemma 3.3] on each interval $\left[0, T_{a}\right]$ we get that $K$ satisfies (b). By [24, Lemma 3.8], we have

$$
\lim _{a \rightarrow \infty} Y_{T_{a}} \leq \xi
$$

On the other hand, taking $\tau=T$ in (5.3), we have $Y_{\alpha} \geq E\left(\xi \mid \mathcal{F}_{\alpha}\right)$. Hence $\lim _{a \rightarrow \infty} Y_{T_{a}} \geq \xi$. From this and (5.4), we deduce that $\lim _{a \rightarrow \infty} Y_{T_{a}}=\xi$. Uniqueness follows from Proposition 4.3.

\subsection{A priori estimates for solutions to one barrier RBSDEs}

We shall need the following additional assumption.

(H5) There exists a process $X$ such that $L \leq X, E \int_{0}^{T} f^{-}\left(r, X_{r}\right) d r<\infty$ and $X$ is a difference of two supermartingales of class (D) on $[0, T]$.

Proposition 5.2 (A priori estimate). Assume that (H1),(H2),(H5) are satisfied and $E|\xi|<$ $\infty$. Then there exists $c>0$ such that for any solution $(Y, M, K)$ of $\underline{\operatorname{RBSDE}}^{T}(\xi, f+d V, L)$ such that $Y$ is of class (D),

$$
\begin{aligned}
\|Y\|_{1}+E \int_{0}^{T}\left|f\left(r, Y_{r}\right)\right| d r+E K_{T} \leq & c\left(\|X\|_{1}+E \int_{0}^{T}|f(r, 0)| d r+E \int_{0}^{T} d|V|_{r}\right. \\
& \left.+E \int_{0}^{T} f^{-}\left(r, X_{r}\right) d r+E \int_{0}^{T} d|C|_{r}\right),
\end{aligned}
$$

where $X_{t}=X_{0}+C_{t}+H_{t}, t \geq 0$, is the Doob-Meyer decomposition of the process $X$ appearing in (H5). Moreover, $M$ is a uniformly integrable martingale and

$$
M_{t}=E\left(\int_{0}^{T} f\left(r, Y_{r}\right) d r+\int_{0}^{T} d V_{r}+\int_{0}^{T} d K_{r} \mid \mathcal{F}_{t}\right)-Y_{0}, \quad t \in[0, T] .
$$

Proof. Let $X$ be as in condition (H5), and $H \in \mathcal{M}_{l o c}, C \in \mathcal{V}_{p}^{1}$ be as in the assertion of the proposition. Observe that

$$
X_{t}=X_{T_{a}}+\int_{t}^{T_{a}} f\left(r, X_{r}\right) d r+\int_{t}^{T_{a}} d V_{r}+\int_{t}^{T_{a}} d \bar{C}_{r}-\int_{t}^{T_{a}} d H_{r}, \quad t \in\left[0, T_{a}\right],
$$

where $\bar{C}_{t}=-\int_{0}^{t} f\left(r, X_{r}\right) d r-V_{t}+C_{t}$. Thus, $(X, H)$ is a solution to $\operatorname{BSDE}^{T}\left(X_{T}, f+d V+d \bar{C}\right)$ (note that $X_{T}$ is well defined by (H5)). By [24, Proposition 2.7] there exists a solution $(\bar{X}, \bar{H})$ of $\mathrm{BSDE}^{T}\left(\xi \vee X_{T}, f+d V^{+}+d \bar{C}^{+}\right)$such that $\bar{X}$ is of class (D). By Proposition 4.3, $\bar{X} \geq X$, so $\bar{X} \geq L$. Observe that the triple $(\bar{X}, \bar{H}, 0)$ is a solution of $\operatorname{RBSDE}^{T}\left(\xi, f+d V^{+}+\right.$ $\left.d \bar{C}^{+}, \bar{X}\right)$. Therefore, by Proposition 4.3, $\bar{X} \geq Y$. By [22, Proposition 4.3],

$$
\left\|Y_{\cdot \wedge T_{a}}\right\|_{1}+E K_{T_{a}} \leq E\left(\left|Y_{T_{a}}\right|+2\|X\|_{1}+\int_{0}^{T}|f(r, 0)| d r+\int_{0}^{T} f^{-}\left(r, \bar{X}_{r}\right) d r+\int_{0}^{T} d|V|_{r}\right) .
$$

Letting $a \rightarrow \infty$ and using the fact that $Y$ is of class (D) on $[0, T]$, we get

$$
\|Y\|_{1}+E K_{T} \leq E\left(|\xi|+2\|X\|_{1}+\int_{0}^{T}|f(r, 0)| d r+\int_{0}^{T} f^{-}\left(r, \bar{X}_{r}\right) d r+\int_{0}^{T} d|V|_{r}\right) .
$$

From this inequality, [24, Theorem 2.9] applied to $(Y, M)$ (as a solution to $\operatorname{BSDE}^{T}(\xi, f+$ $d K+d V)$ ) and $(\bar{X}, \bar{H})$, we infer the desired inequality. The second part of the assertion now follows easily from equation (d) of Definition 4.1 . 


\subsection{Existence results for one barrier RBSDEs}

Proposition 5.3 (Existence: Lipschitz continuous driver case). Assume that (H1), (H2), (H4) are satisfied, $E|\xi|<\infty$, and there is a Borel measurable function $\lambda:[0, \infty) \rightarrow \mathbb{R}^{+}$ such that $\int_{0}^{\infty} \lambda(r) d r<\infty$ and

$$
\left|f\left(t, y_{1}\right)-f\left(t, y_{2}\right)\right| \leq \lambda(t)\left|y_{1}-y_{2}\right|, \quad t \in[0, T], y_{1}, y_{2} \in \mathbb{R} .
$$

Then there exists a unique solution $(Y, M, K)$ of $\underline{\operatorname{RBSDE}}^{T}(\xi, f+d V, L)$ such that $Y$ is of class (D).

Proof. Let $\left(Y^{0}, M^{0}\right)$ be a solution of $\operatorname{BSDE}^{T}(\xi, f+d V)$ such that $Y^{0}$ is of class (D). The existence of the solution follows from [24, Proposition 2.7]. Observe that by (5.5) and (H1), for any process $X$ of class (D), $E \int_{0}^{T}\left|f\left(r, X_{r}\right)\right| d r<\infty$. Therefore, by Proposition 5.1 , for each $n \geq 1$, there exists a solution $\left(Y^{n}, M^{n}, K^{n}\right)$ of $\underline{\operatorname{RBSDE}}^{T}\left(\xi, f_{Y^{n-1}}+d V, L\right)$ such that $Y^{n}$ is of class (D). Moreover,

$$
Y_{\alpha}^{n}=\operatorname{esssup}_{\tau \in \mathcal{T}_{\alpha}} E\left(\int_{\alpha}^{\tau} f\left(r, Y_{r}^{n-1}\right) d r+\int_{\alpha}^{\tau} d V_{r}+L_{\tau} \mathbf{1}_{\{\tau<T\}}+\xi \mathbf{1}_{\{\tau=T\}} \mid \mathcal{F}_{\alpha}\right), \quad \alpha \in \mathcal{T} .
$$

By Proposition 4.3,

$$
Y^{0} \leq Y^{2} \leq Y^{4} \leq Y^{6} \leq \cdots \leq Y^{5} \leq Y^{3} \leq Y^{1} .
$$

Set $\bar{Y}=\lim _{n \rightarrow \infty} Y^{2 n}, \underline{Y}=\lim _{n \rightarrow \infty} Y^{2 n+1}$. Clearly $\bar{Y}, \underline{Y}$ are of class (D). By (5.5)-(5.7) and the Lebesgue dominated convergence theorem

$$
\bar{Y}_{\alpha}=\operatorname{esssup}_{\tau \in \mathcal{T}_{\alpha}} E\left(\int_{\alpha}^{\tau} f\left(r, \underline{Y}_{r}\right) d r+\int_{\alpha}^{\tau} d V_{r}+L_{\tau} \mathbf{1}_{\{\tau<T\}}+\xi \mathbf{1}_{\{\tau=T\}} \mid \mathcal{F}_{\alpha}\right), \quad \alpha \in \mathcal{T},
$$

and

$$
\underline{Y}_{\alpha}=\operatorname{esssup}_{\tau \in \mathcal{T}_{\alpha}} E\left(\int_{\alpha}^{\tau} f\left(r, \bar{Y}_{r}\right) d r+\int_{\alpha}^{\tau} d V_{r}+L_{\tau} \mathbf{1}_{\{\tau<T\}}+\xi \mathbf{1}_{\{\tau=T\}} \mid \mathcal{F}_{\alpha}\right), \quad \alpha \in \mathcal{T} .
$$

By Propositions 5.1, 5.2 there exist $\bar{K}, \underline{K} \in \mathcal{V}_{p}^{+, 1}$ and $\bar{M}, \underline{M} \in \mathcal{M}$ such that $(\bar{Y}, \bar{M}, \bar{K})$ is

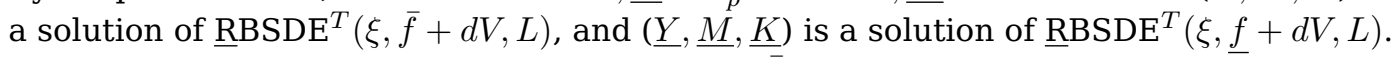
The proof will be complete once we prove that $\bar{Y}_{t}=\underline{Y}_{t}$ for a.e. $t \geq 0$. By [25, Corollary A.5] and (5.5), for any stopping time $\sigma \in \mathcal{T}$,

$$
\begin{aligned}
E\left|\bar{Y}_{\sigma}-\underline{Y}_{\sigma}\right| \leq & E \int_{\sigma}^{T} \lambda(r)\left|\bar{Y}_{r}-\underline{Y}_{r}\right| d r+E \int_{\sigma}^{T} \operatorname{sgn}\left(\bar{Y}_{r-}-\underline{Y}_{r-}\right) d\left(\bar{K}_{r}-\underline{K}_{r}\right)^{*} \\
& +E \sum_{\sigma \leq r<T} \operatorname{sgn}\left(\bar{Y}_{r}-\underline{Y}_{r}\right) \Delta^{+}\left(\bar{K}_{r}-\underline{K}_{r}\right) .
\end{aligned}
$$

By the minimality condition for $\bar{K}, \underline{K}$ and the fact that $L \leq \bar{Y}, \underline{Y}$, we have

$$
\operatorname{sgn}\left(\bar{Y}_{r}>\underline{Y}_{r}\right) d\left(\bar{K}_{r}-\underline{K}_{r}\right)^{*} \leq \mathbf{1}_{\left\{\bar{Y}_{r}>L_{r}\right\}} d \bar{K}_{r}^{*}+\mathbf{1}_{\left\{\underline{Y}_{r}>L_{r}\right\}} d \underline{K}_{r}^{*}=0,
$$

and

$$
\operatorname{sgn}\left(\bar{Y}_{r}>\underline{Y}_{r}\right) \Delta^{+}\left(\bar{K}_{r}-\underline{K}_{r}\right) \leq \mathbf{1}_{\left\{\bar{Y}_{r}>L_{r}\right\}} \Delta^{+} \bar{K}_{r}+\mathbf{1}_{\left\{\underline{Y}_{r}>L_{r}\right\}} \Delta^{+} \underline{K}_{r}=0 .
$$

By (5.8)-(5.10)

$$
E\left|\bar{Y}_{\sigma}-\underline{Y}_{\sigma}\right| \leq \int_{\sigma}^{\infty} \lambda(r) E\left|\bar{Y}_{r}-\underline{Y}_{r}\right| d r, \quad \sigma \in \mathcal{T}
$$

Applying now Gronwall's lemma yields the result. 
Proposition 5.4 (Existence: bounded from below driver case). Assume that (H1)-(H4) are satisfied, $E|\xi|<\infty$, and there exists a progressively measurable process $g$ such that $E \int_{0}^{T}|g(r)| d r<\infty$ and $f(t, y) \geq g(t), t \in[0, T], y \in \mathbb{R}$. Then there exists a unique solution $(Y, M, K)$ of $\underline{\operatorname{RBSDE}}^{T}(\xi, f+d V, L)$ such that $Y$ is of class (D). Moreover, $K \in \mathcal{V}_{p}^{1,+}$ and $M \in \mathcal{M}$.

Proof. For each $n \geq 1$, we let

$$
f_{n}(r, y)=c_{n}(r) \inf _{x \in \mathbb{R}}\{f(r, x)+n|y-x|\},
$$

where $c_{n}:[0, \infty) \rightarrow[0,1]$ is a Borel measurable function such that $\int_{0}^{\infty} c_{n}(r) d r<\infty$, and $c_{n}(r) \nearrow 1$ as $n \rightarrow \infty$ for every $r \geq 0$. It is easy to check that for each $n \geq 1$ the hypotheses (H1), (H2) and (H4) are satisfied for data $\left(\xi, f_{n}, V\right)$. Moreover, for any $t \in[0, T]$ and $y_{1}, y_{2} \in \mathbb{R}$,

$$
\left|f_{n}\left(t, y_{1}\right)-f_{n}\left(t, y_{2}\right)\right| \leq c_{n}(t) n\left|y_{1}-y_{2}\right| \text {. }
$$

Therefore, by Proposition 5.3, there exists a solution $\left(Y^{n}, M^{n}, K^{n}\right)$ of $\underline{\operatorname{RBSDE}}^{T}\left(\xi, f_{n}+\right.$ $d V, L)$ such that $Y^{n}$ is of class (D). Observe that for each $n \geq 1, f_{n} \leq f_{n+1}$. Thus, by Proposition 4.3, we have $Y^{n} \leq Y^{n+1}, n \geq 1$. Set $Y=\sup _{n \geq 1} Y^{n}$. We shall prove that $Y$ is of class (D) and $\lim _{a \rightarrow \infty} Y_{T_{a}}=\xi$. By Propositions 5.1,5.2 and the assumptions on $f$ there exists a solution $(X, N, A)$ of $\operatorname{RBSDE}^{T}(\xi, 0, L)$ such that $X$ is of class (D), $E \int_{0}^{T}\left|f\left(r, X_{r}\right)\right| d r<\infty$, and $E A_{T}<\infty$. Observe that $(X, N)$ is a solution of $\operatorname{BSDE}^{T}(\xi, f+$ $d V+d \bar{A})$, where $\bar{A}_{t}=-\int_{0}^{t} f\left(r, X_{r}\right) d r-V_{t}+A_{t}$. By [24, Proposition 2.7] there exists a solution $(\bar{X}, \bar{H})$ of $\operatorname{BSDE}^{T}\left(\xi, f+d V^{+}+d \bar{A}^{+}\right)$such that $\bar{X}$ is of class (D). By Proposition 4.3, $\bar{X} \geq X$, so $\bar{X} \geq L$. Clearly, the triple $(\bar{X}, \bar{H}, 0)$ also is a solution of $\operatorname{RBSDE}^{T}(\xi, f+$ $\left.d V^{+}+d \bar{A}^{+}, \bar{X}\right)$. Therefore, by Proposition $4.3, \bar{X} \geq Y^{n}, n \geq 1$. Summarizing, we have

$$
Y^{1} \leq Y^{n} \leq \bar{X}, \quad n \geq 1
$$

Thus, $Y$ is of class (D) and $\lim _{a \rightarrow \infty} Y_{T_{a}}=\xi$. By (H3), $f_{n}\left(r, Y_{r}^{n}\right) \rightarrow f\left(r, Y_{r}\right)$ as $n \rightarrow \infty$. Since $f_{n} \leq f_{n+1}$ we infer from (H2), (5.11) and the assumption on $f$ that

$$
g(r) \leq f_{n}\left(r, Y_{r}^{n}\right) \leq f\left(r, Y_{r}^{1}\right)
$$

Set

$$
\tau_{k}=\inf \left\{t \geq 0 ; \int_{0}^{t}\left|f\left(r, Y_{r}^{1}\right)\right| d r \geq k\right\} \wedge T .
$$

By (H4), $\left\{\tau_{k}\right\}$ is a chain on $[0, T]$. Observe that the triple $\left(Y^{n}, M^{n}, K^{n}\right)$ is a solution of $\underline{\operatorname{RBSDE}}^{\tau_{k}}\left(Y_{\tau_{k}}^{n}, f_{n}+d V, L\right)$. Hence, by Proposition 5.1, for every $\sigma \in \mathcal{T}_{\tau_{k}}$,

$$
Y_{\sigma}^{n}=\operatorname{esssup}_{\tau \in \mathcal{T}_{\sigma, \tau_{k}}} E\left(\int_{\sigma}^{\tau} f_{n}\left(r, Y_{r}^{n}\right) d r+\int_{\sigma}^{\tau} d V_{r}+L_{\tau} \mathbf{1}_{\left\{\tau<\tau_{k}\right\}}+Y_{\tau_{k}}^{n} \mathbf{1}_{\left\{\tau=\tau_{k}\right\}} \mid \mathcal{F}_{\sigma}\right) .
$$

By the definition of $\tau_{k}$ and (5.12),

$$
E \int_{0}^{\tau_{k}}\left|f_{n}\left(r, Y_{r}^{n}\right)-f\left(r, Y_{r}\right)\right| d r \rightarrow 0
$$

as $n \rightarrow \infty$. By (5.11), (5.13), (5.14) and [25, Lemma 3.19],

$$
Y_{\sigma}=\operatorname{essips}_{\tau \in \mathcal{T}_{\sigma, \tau_{k}}} E\left(\int_{\sigma}^{\tau} f\left(r, Y_{r}\right) d r+\int_{\sigma}^{\tau} d V_{r}+L_{\tau} \mathbf{1}_{\left\{\tau<\tau_{k}\right\}}+Y_{\tau_{k}} \mathbf{1}_{\left\{\tau=\tau_{k}\right\}} \mid \mathcal{F}_{\sigma}\right)
$$

for $\sigma \in \mathcal{T}^{\tau_{k}}$. By Proposition 5.1 there exist $K^{k} \in \mathcal{V}_{p}^{+}$and $M^{k} \in \mathcal{M}_{\text {loc }}$ such that $\left(Y, M^{k}, K^{k}\right)$ is a solution of $\underline{\operatorname{RBSDE}}^{\tau_{k}}\left(Y_{\tau_{k}}, f+d V, L\right)$. By uniqueness, for any $a \geq 0, K_{t}^{k}=K_{t}^{k+1}$ and 
$M_{t}^{k}=M_{t}^{k+1}, t \in\left[0, \tau_{k} \wedge a\right]$. Therefore, we may define processes $K$ and $M$ on $[0, T] \backslash\{\infty\}$ by putting $K_{t}=K_{t}^{k}, M_{t}=M_{t}^{k}$ on $\left[0, \tau_{k} \wedge a\right], a \geq 0$. Since $\left\{\tau_{k}\right\}$ is a chain on $[0, T]$, we get that $(Y, M, K)$ is a solution of $\operatorname{RBSDE}^{T}(\xi, f+d V, L)$. Uniqueness follows from Proposition 4.3. The second assertion of the proposition follows from Proposition 5.2 since $f^{-} \leq|g|$.

Theorem 5.5 (The existence result for one barrier RBSDE). Assume that (H1)-(H4) are satisfied and $E|\xi|<\infty$. Then there exists a solution $(Y, M, K)$ of $\underline{\operatorname{RBSDE}}^{T}(\xi, f+d V, L)$ such that $Y$ is of class (D).

Proof. Consider a strictly positive Borel measurable function $g:[0, \infty) \rightarrow \mathbb{R}$ such that $\int_{0}^{\infty} g(r) d r<\infty$. For each $n \geq 1$, we let

$$
f_{n}(r, y)=f(r, y) \vee(-n \cdot g(r)), \quad y \in \mathbb{R} .
$$

By Theorem 5.4 for each $n \geq 1$ there exists a solution $\left(Y^{n}, M^{n}, K^{n}\right)$ of $\underline{\operatorname{RBSDE}}^{T}\left(\xi, f_{n}+\right.$ $d V, L)$ such that $Y^{n}$ is of class (D). Since $f_{n} \geq f_{n+1}$, we have by Proposition $4.3, Y^{n} \geq$ $Y^{n+1}$. Set $Y=\inf _{n \geq 1} Y^{n}$. By [24, Proposition 2.7] there exists a solution $(X, H)$ to $\operatorname{BSDE}^{T}(\xi, f+d V)$ such that $X$ is of class (D). By Proposition 4.3,

$$
X \leq Y^{n} \leq Y^{1}, \quad n \geq 1
$$

thus $Y$ is of class (D) and $\lim _{a \rightarrow \infty} Y_{T_{a}}=\xi$. By (H3), $f_{n}\left(r, Y_{r}^{n}\right) \rightarrow f\left(r, Y_{r}\right)$ as $n \rightarrow \infty$. Moreover, since $f_{n} \geq f_{n+1}$, it follows from (H2) and (5.15) that

$$
f\left(r, Y_{r}^{1}\right) \leq f_{n}\left(r, Y_{r}^{n}\right) \leq f_{1}\left(r, X_{r}\right)
$$

Set

$$
\tau_{k}=\inf \left\{t \geq 0: \int_{0}^{t}\left|f\left(r, Y_{r}^{1}\right)\right|+\left|f_{1}\left(r, X_{r}\right)\right| d r \geq k\right\} \wedge T .
$$

Then, by (H4), $\left\{\tau_{k}\right\}$ is a chain on $[0, T]$. Observe that $\left(Y^{n}, M^{n}, K^{n}\right)$ is a solution to $\underline{\operatorname{RBSDE}}^{\tau_{k}}\left(Y_{\tau_{k}}^{n}, f_{n}+d V, L\right)$. Therefore, by Proposition 5.1, for every $\sigma \in \mathcal{T}^{\tau_{k}}$,

$$
Y_{\sigma}^{n}=\operatorname{esssup}_{\tau \in \mathcal{T}_{\sigma, \tau_{k}}} E\left(\int_{\sigma}^{\tau} f\left(r, Y_{r}^{n}\right) d r+\int_{\sigma}^{\tau} d V_{r}+L_{\tau} \mathbf{1}_{\left\{\tau<\tau_{k}\right\}}+Y_{\tau_{k}}^{n} \mathbf{1}_{\left\{\tau=\tau_{k}\right\}} \mid \mathcal{F}_{\sigma}\right)
$$

By the definition of $\tau_{k}$ and (5.16),

$$
E \int_{0}^{\tau_{k}}\left|f_{n}\left(r, Y_{r}^{n}\right)-f\left(r, Y_{r}\right)\right| d r \rightarrow 0
$$

Now, repeating step by step the reasoning following (5.14), we get the desired result.

Remark 5.6. Instead of condition (H1) on $f$ one can consider the following seemingly more general condition: there exists a process $S$ which is a difference of two supermartingales of class (D) such that $E \int_{0}^{T}\left|f\left(r, S_{r}\right)\right| d r<\infty$. However, this hypothesis, in fact, would not affect the generality of the results of the paper. Indeed, let $S=S_{0}+C+N$ by the Doob-Meyer decomposition of $S\left(C \in \mathcal{V}_{p}^{+, 1}, N \in \mathcal{M}\right)$. Observe that $(Y, M, K)$ is a solution of $\underset{\tilde{R} B S D E}{ }{ }^{T}(\xi, f+d V, L)$ if and only if $(\tilde{Y}, \tilde{M}, K)$ is a solution to $\underline{\operatorname{RBSDE}}^{T}(\tilde{\xi}, \tilde{f}+d \tilde{V}, \tilde{L})$, where $\tilde{\tilde{Y}}=Y-S, \tilde{M}=M-N, \tilde{\xi}=\xi-S_{T}, \tilde{f}(r, y)=f\left(r, y+S_{r}\right), \tilde{V}=V+C$ and $\tilde{L}=L-S$. 


\subsection{Optimal stopping problem with nonlinear expectation}

Repeating step by step the proofs of the results of Section 8 in [15] and [15, Theorem 6.1], with using Proposition 3.4 and Proposition 4.3, we get the following result.

Lemma 5.7. Assume that (H1)-(H4) are satisfied and $E|\xi|<\infty$. For $\alpha \in \mathcal{T}$, let

$$
Y(\alpha):=\operatorname{essiup}_{\tau \in \mathcal{T}} \mathcal{E}_{\alpha, \tau}^{f}\left(L_{\tau}^{\xi}\right)
$$

where $L_{t}^{\xi}=L_{t} \mathbf{1}_{t<T}+\xi \mathbf{1}_{t=T}$. Then

(i) There exists an optional process $Y$ which aggregates the family $(Y(\alpha))_{\alpha \in \mathcal{T}}$, that is $Y_{\alpha}=Y(\alpha)$ for every $\alpha \in \mathcal{T}$.

(ii) $Y$ is the smallest $\mathcal{E}^{f}$-supermartingale majorizing $L^{\xi}$,

(iii) If $L$ is u.s.c. from the right, then $Y$ coincides with the first component of the solution to $\operatorname{RBSDE}^{T}(\xi, f, L)$.

Theorem 5.8 (Nonlinear Snell envelope representation). Assume that (H1)-(H4) are satisfied and $E|\xi|<\infty$. Let $(Y, M, K)$ be a solution of $\operatorname{RBSDE}^{T}(\xi, f+d V, L)$ such that $Y$ is of class (D). Then for every $\alpha \in \mathcal{T}$,

$$
Y_{\alpha}=\operatorname{ess~sup}_{\tau \in \mathcal{T}_{\alpha}} \mathcal{E}_{\alpha, \tau}^{f}\left(\int_{\alpha}^{\tau} d V_{r}+L_{\tau} \mathbf{1}_{\tau<T}+\xi \mathbf{1}_{\tau=T}\right) .
$$

Proof. Without loss of generality we may assume that $V \equiv 0$ (cf. the beginning of the proof of Proposition 5.1). By Proposition 3.4(i), $Y$ is an $\mathcal{E}^{f}$-supermartingale. Of course, $Y \geq L^{\xi}$. Let $Y^{\prime}$ be an $\mathcal{E}^{f}$-supermartingale such that $Y^{\prime} \geq L^{\xi}$ and $(\bar{Y}, \bar{M}, \bar{K})$ be a solution to $\operatorname{RBSDE}^{T}\left(Y_{T}^{\prime}, f, Y^{\prime}\right)$. Then, by Proposition 4.3, $\bar{Y} \geq Y$. On the other hand, since $Y^{\prime}$ is an $\mathcal{E}^{f}$-supermartingale, $Y_{\alpha}^{\prime}=\operatorname{ess}_{\sup } \operatorname{su}_{\tau} \mathcal{E}_{\alpha, \tau}^{f}\left(Y_{\tau}^{\prime}\right)$. Therefore, by Lemma 5.7(iii) and Proposition 4.3, $\bar{Y}=Y^{\prime}$ (since $Y^{\prime}$ is u.s.c. from the right as an $\mathcal{E}^{f}$-supermartingale). Thus $Y^{\prime} \leq Y$, which implies that $Y$ is the smallest $\mathcal{E}^{f}$-supermartingale majorizing $L^{\xi}$. This when combined with Lemma 5.7(ii) gives the desired result.

\section{Existence results for RBSDEs with two barriers and Dynkin games}

In this section, we shall prove existence results for reflected BSDEs with two optional barriers that satisfy the following Mokobodzki's condition:

(H6) There exists a special semimartingale $X$ such that $L \leq X \leq U$.

First, we focus on the existence result which asserts additionally (see Theorem 6.1) that variations of $\int_{0}^{\cdot} f\left(r, Y_{r}\right) d r$ and $R$ on $[0, T]$ are integrable. For this we shall need a stronger version of (H6) of the following form:

(H6*) There exists a process $X$ being the difference of two supermartingales of class (D) on $[0, T]$ such that $L \leq X \leq U$ and $E \int_{0}^{T}\left|f\left(r, X_{r}\right)\right| d r<\infty$.

We call (H6*) the strong Mokobodzki's condition. Observe that (H6*) is also a necessary condition for the mentioned integrability of $\int_{0}^{\cdot} f\left(r, Y_{r}\right) d r$ and $R$. To give an existence result under general condition (H6), we first prove stability results for RBSDEs (see Subsection 6.2), i.e. results concerning convergence of $Y^{n}$ to $Y$ when $\left(\xi_{n}, f_{n}, L^{n}, U^{n}\right)$ converges to $(\xi, f, L, U)$. In this regard, we prove Corollary 6.5 and Proposition 6.7. Both results have different limitations. To apply Corollary 6.5, we need integrability of $\int_{0}^{T}\left|f_{n}\left(r, Y_{r}\right)\right| d r$ and $\int_{0}^{T}\left|f\left(r, Y_{r}\right)\right| d r$ (see Remark 6.6), while applying 
Proposition 6.7 requires monotone convergence of data. Finally, in Subsection 6.3, we prove the main result (Theorem 6.8) of this section, i.e. the existence result for RBSDEs under (H1)-(H4), (H6). We close the paper with an observation that the proof of Theorem 6.8 applies even if we dispense with integrability assumptions on $f$ and $V$ included in (H1). Therefore, we get the existence result (Theorem 6.9) under (H2)-(H4), (H6). This may seem strange, since (H1) is necessary for the existence of solutions to BSDEs with no reflection. However, a moment's consideration on the definition of a solution to reflected BSDE makes it clear that additional condition on $Y$, as compared to non-reflected BSDEs, of being between the barriers prevents $Y$ from blowing up, and hence allows one to consider weaker assumptions.

\subsection{Existence for RBSDEs under strong Mokobodzki's condition}

Theorem 6.1 (Existence of integrable solutions of two barriers RBSDEs). Assume that (H1)-(H4), (H6*) are satisfied. Then there exists a unique solution $(Y, M, R)$ of the problem $\operatorname{RBSDE}^{T}(\xi, f+d V, L, U)$ such that $Y$ is of class (D). Moreover, $R \in \mathcal{V}_{p}^{1}, M$ is a martingale of class (D) on $[0, T]$, and

$$
E \int_{0}^{T}\left|f\left(r, Y_{r}\right)\right| d r<\infty
$$

Proof. Let $\left(Y^{1,0}, M^{1,0}\right)$ be a solution of $\operatorname{BSDE}^{T}(\xi, f+d V)$ such that $Y^{1,0}$ is of class (D), and let $\left(Y^{2,0}, Z^{2,0}\right)=(0,0)$. Next, for each $n \geq 1$, let $\left(Y^{1, n}, M^{1, n}, K^{1, n}\right)$ be a solution of $\underline{\operatorname{RBSDE}}^{T}\left(\xi, f_{n}+d V, L+Y^{2, n-1}\right)$ with

$$
f_{n}(r, y)=f\left(r, y-Y_{r}^{2, n-1}\right),
$$

and let $\left(Y^{2, n}, M^{2, n}, K^{2, n}\right)$ be a solution of $\underline{\operatorname{RBSDE}}^{T}\left(0,0, Y^{1, n-1}-U\right)$ such that $Y^{1, n}, Y^{2, n}$ are of class (D). In both cases the existence of required solutions follows from Theorem 5.5 (see also Remark 5.6). We shall prove by induction that the sequences $\left(Y^{1, n}\right)_{n \geq 0}$, $\left(Y^{2, n}\right)_{n \geq 0}$ are non-decreasing. Clearly $Y^{1,1} \geq Y^{1,0}$ and $Y^{2,1} \geq Y^{2,0}$. Suppose that for fixed $n \in \mathbb{N}, Y^{1, n} \geq Y^{1, n-1}$ and $Y^{2, n} \geq Y^{2, n-1}$. Using this and (H2) we infer that $f_{n+1} \geq f_{n}$ and $L+Y^{2, n} \geq L+Y^{2, n-1}$. Thus, by Proposition 4.3, $Y^{1, n+1} \geq Y^{1, n}$. By a similar argument, $Y^{2, n+1} \geq Y^{2, n}$. Consequently, $\left(Y^{1, n}\right)_{n \geq 0}$ and $\left(Y^{2, n}\right)_{n \geq 0}$ are non-decreasing. Next we show that $Y^{1}:=\sup _{n \geq 1} Y^{1, n}$ and $Y^{2}:=\sup _{n \geq 1} Y^{2, n}$ are of class (D). Let $X$ be as in (H6*). Then there exist processes $H \in \mathcal{M}$ and $C \in \mathcal{V}_{p}^{1}$ such that for every $a \geq 0$,

$$
X_{t}=X_{T_{a}}+\int_{t}^{T_{a}} d C_{r}-\int_{t}^{T_{a}} d H_{r}, \quad t \in\left[0, T_{a}\right] .
$$

This equation may be rewritten in the form

$$
X_{t}=X_{T_{a}}+\int_{t}^{T_{a}} f\left(r, X_{r}\right) d r+\int_{t}^{T_{a}} d V_{r}+\int_{t}^{T_{a}} d \bar{C}_{r}-\int_{t}^{T_{a}} d H_{r}
$$

where $\bar{C}_{t}=-\int_{0}^{t} f\left(r, X_{r}\right) d r-V_{t}+C_{t}$. Let $\left(X^{2}, H^{2}\right)$ be a solution of $\operatorname{BSDE}^{T}\left(0, d \bar{C}^{-}\right)$and $\left(X^{1}, H^{1}\right)$ be a solution of $\operatorname{BSDE}^{T}\left(X_{T} \vee \xi, \tilde{f}+d V+d \bar{C}^{+}\right)$such that $X^{1}, X^{2}$ are of class (D) (cf. Theorem 5.5), where $\tilde{f}(r, x)=f\left(r, x-X_{r}^{2}\right)$. Observe that $X=X^{1}-X^{2}$. This implies that $X^{1} \geq L+X^{2}$ and $X^{2} \geq X^{1}-U$. Therefore, $\left(X^{1}, H^{1}, 0\right)$ is a solution of $\underline{\operatorname{RBSDE}^{T}}\left(X_{T} \vee \xi, \overline{\tilde{f}}+d V+d \bar{C}^{+}, L+X^{2}\right)$ and $\left(X^{2}, H^{2}, 0\right)$ is a solution of $\operatorname{RBSDE}^{T}\left(0, d \bar{C}^{-}\right.$, $\left.X^{1}-U\right)$. We shall prove by induction that for each $n \in \mathbb{N}, X^{1} \geq Y^{1, n}$ and $X^{2} \geq Y^{2, n}$. Let $n=0$. Since $X^{2} \geq 0$, it follows from (H2) that $\tilde{f} \geq f$. Thus, by Proposition 4.3, $X^{1} \geq Y^{1,0}$. Since $Y^{2,0}=0$, we have $X^{2} \geq Y^{2,0}$. Suppose that for fixed $n \in \mathbb{N}, X^{1} \geq Y^{1, n}$ and $X^{2} \geq Y^{2, n}$. Then $L+X^{2} \geq L+Y^{2, n}$ and $X^{1}-U \geq Y^{1, n}-U$, and by (H2) we have 
$\tilde{f} \geq f_{n+1}$. Consequently, by Proposition $4.3, X^{1} \geq Y^{1, n+1}, X^{2} \geq Y^{2, n+1}$, the induction is complete. Summarizing, we have

$$
Y^{1,0} \leq Y^{1, n} \leq X^{1}, \quad 0=Y^{2,0} \leq Y^{2, n} \leq X^{2}, \quad n \geq 0 .
$$

Therefore, $Y^{1}, Y^{2}$ are of class (D) and

$$
\xi \leq \lim _{a \rightarrow \infty} Y_{T_{a}}^{1} \quad \lim _{a \rightarrow \infty} Y_{T_{a}}^{2}=0 .
$$

We shall show that there exist $M^{1}, M^{2} \in \mathcal{M}_{\text {loc }}, K^{1}, K^{2} \in \mathcal{V}^{+}$such that $\left(Y^{1}, M^{1}, K^{1}\right)$ is a solution of $\underline{\operatorname{RBSDE}}^{T}\left(\xi, \hat{f}+d V, L+Y^{2}\right)$ with

$$
\hat{f}(r, y)=f\left(r, y-Y_{r}^{2}\right)
$$

and $\left(Y^{2}, M^{2}, K^{2}\right)$ is a solution of $\underline{\operatorname{RBSDE}}^{T}\left(0,0, Y^{1}-U\right)$. By (H3),

$$
f\left(r, Y_{r}^{1, n}-Y_{r}^{2, n-1}\right) \rightarrow f\left(r, Y_{r}^{1}-Y_{r}^{2}\right), \quad \text { as } n \rightarrow \infty .
$$

Moreover, by (H2) and (6.2),

$$
f\left(r, X_{r}^{1}\right) \leq f\left(r, Y_{r}^{1, n}-Y_{r}^{2, n-1}\right) \leq f\left(r, Y_{r}^{1,0}-X_{r}^{2}\right) .
$$

Set

$$
\tau_{k}=\inf \left\{t \geq 0: \int_{0}^{t}\left|f\left(r, Y_{r}^{1,0}-X_{r}^{2}\right)\right|+\left|f\left(r, X_{r}^{1}\right)\right| d r \geq k\right\} \wedge T .
$$

By (H4), $\left\{\tau_{k}\right\}$ is a chain on $[0, T]$. By Proposition 5.1, and (5.2) for every $\sigma \in \mathcal{T}^{\tau_{k}}$,

$$
\begin{aligned}
Y_{\sigma}^{1, n}= & \operatorname{esssup}_{\tau \in \mathcal{T}_{\sigma, \tau_{k}}} E\left(\int_{\sigma}^{\tau} f\left(r, Y_{r}^{1, n}-Y_{r}^{2, n-1}\right) d r+\int_{\sigma}^{\tau} d V_{r}\right. \\
& \left.+\left(L_{\tau}+Y_{\tau}^{2, n-1}\right) \mathbf{1}_{\left\{\tau<\tau_{k}\right\}}+Y_{\tau_{k}}^{1, n} \mathbf{1}_{\left\{\tau=\tau_{k}\right\}} \mid \mathcal{F}_{\sigma}\right) .
\end{aligned}
$$

By the definition of $\tau_{k}$, (H4), (6.4) and (6.5),

$$
E \int_{0}^{\tau_{k}}\left|f\left(r, Y_{r}^{1, n}-Y_{r}^{2, n-1}\right)-f\left(r, Y_{r}^{1}-Y_{r}^{2}\right)\right| d r \rightarrow 0, \quad \text { as } \quad n \rightarrow \infty .
$$

By (6.2), (6.6), (6.7) and [25, Lemma 3.19],

$$
\begin{aligned}
Y_{\sigma}^{1}= & \operatorname{esssup}_{\tau \in \mathcal{T}_{\sigma, \tau_{k}}} E\left(\int_{\sigma}^{\tau} f\left(r, Y_{r}^{1}-Y_{r}^{2}\right) d r+\int_{\sigma}^{\tau} d V_{r}\right. \\
& \left.+\left(L_{\tau}+Y_{\tau}^{2}\right) \mathbf{1}_{\left\{\tau<\tau_{k}\right\}}+Y_{\tau_{k}}^{1} \mathbf{1}_{\left\{\tau=\tau_{k}\right\}} \mid \mathcal{F}_{\sigma}\right) .
\end{aligned}
$$

for every $\sigma \in \mathcal{T}^{\tau_{k}}$. By [24, Lemma 3.8], $\lim _{a \rightarrow \infty} Y_{\tau_{k} \wedge a}^{1} \leq Y_{\tau_{k}}^{1}$. From this, since $\left\{\tau_{k}\right\}$ is a chain, we infer that $\lim _{a \rightarrow \infty} Y_{T_{a}}^{1} \leq \xi$. Consequently, by (6.3), we get

$$
\lim _{a \rightarrow \infty} Y_{T_{a}}=\xi \text {. }
$$

Next, by Proposition 5.1, for all $n \geq 1$ and $\sigma \in \mathcal{T}$,

$$
Y_{\sigma}^{2, n}=\operatorname{esssup}_{\tau \in \mathcal{T}_{\sigma}} E\left(\left(Y_{\tau}^{1, n-1}-U_{\tau}\right) \mathbf{1}_{\{\tau<T\}} \mid \mathcal{F}_{\sigma}\right) .
$$

Letting $n \rightarrow \infty$ and using (6.2) we get

$$
Y_{\sigma}^{2}=\operatorname{ess~sup}_{\tau \in \mathcal{T}_{\sigma}} E\left(\left(Y_{\tau}^{1}-U_{\tau}\right) \mathbf{1}_{\{\tau<T\}} \mid \mathcal{F}_{\sigma}\right) .
$$


By Proposition 5.1, for any $k \geq 0$, there exist $K^{k} \in \mathcal{V}_{p}^{+}$and $M^{k} \in \mathcal{M}_{\text {loc }}$ such that $\left(Y^{1}, M^{1, k}, K^{1, k}\right)$ is a solution of $\operatorname{RBSDE}^{\tau_{k}}\left(Y_{\tau_{k}}^{1}, \hat{f}+d V, L+Y^{2}\right)$. By the uniqueness argument, $K_{t}^{1, k}=K_{t}^{1, k+1}, M_{t}^{1, k}=M_{t}^{1, k+1}, t \in\left[0, \tau_{k} \wedge a\right], a \geq 0$. Therefore, since $\left\{\tau_{k}\right\}$ is a chain, we may define processes $M^{1}$ and $K^{1}$ on $[0, T] \backslash\{\infty\}$ by putting $M_{t}^{1}=M_{t}^{1, k}, K_{t}^{1}=K_{t}^{1, k}$, $t \in\left[0, \tau_{k} \wedge a\right], a \geq 0$. Clearly, the triple $\left(Y^{1}, M^{1}, K^{1}\right)$ is a solution of $\operatorname{RBSDE}^{T}(\xi, \hat{f}+d V, L+$ $\left.Y^{2}\right)$. By Proposition 5.1 again, there exist $K^{2} \in \mathcal{V}_{p}^{+}, M^{2} \in \mathcal{M}_{\text {loc }}$ such that the triple $\left(Y^{2}, M^{2}, K^{2}\right)$ is a solution of $\operatorname{RBSDE}^{T}\left(0,0, Y^{1}-U\right)$. Write $Y=Y^{1}-Y^{2}, M=M^{1}-M^{2}$, $R=K^{1}-K^{2}$. We shall show that $(Y, M, R)$ is a solution of $\operatorname{RBSDE}^{T}(\xi, f+d V, L, U)$. Directly from the definition of a solution to $\underline{\operatorname{RBSDE}}^{T}\left(0,0, Y^{1}-U\right)$ and $\underline{\operatorname{RBSDE}}^{T}\left(\xi, \hat{f}+d V, L+Y^{2}\right)$ (cf. Definition 4.1), we deduce that $Y$ is of class (D), $\lim _{a \rightarrow \infty} Y_{T_{a}}=\xi, L \leq Y \leq U$, and for any $a \geq 0$,

$$
Y_{t}=Y_{T_{a}}+\int_{t}^{T_{a}} f\left(r, Y_{r}\right) d r+\int_{t}^{T_{a}} d V_{r}+\int_{t}^{T_{a}} d R_{r}-\int_{t}^{T_{a}} d M_{r}, \quad t \in\left[0, T_{a}\right] .
$$

Therefore, what is left is to prove that $R$ satisfies the minimality condition (b) of Definition 4.2. For this observe that for any $a \geq 0$,

$$
\begin{aligned}
& \int_{0}^{T_{a}}\left(Y_{r-}-\vec{L}_{r}\right) d R_{r}^{+, *}+\sum_{0 \leq r<T_{a}}\left(Y_{r}-L_{r}\right) \Delta^{+} R_{r}^{+} \\
& \quad \leq \int_{0}^{T_{a}}\left(Y_{r-}^{1}-\overrightarrow{L_{r}+Y_{r}^{2}}\right) d K_{r}^{1, *}+\sum_{0 \leq r<T_{a}}\left(Y_{r}^{1}-\left(L_{r}+Y_{r}^{2}\right)\right) \Delta^{+} K_{r}^{1}=0
\end{aligned}
$$

and

$$
\begin{aligned}
& \int_{0}^{T_{a}}\left(\underline{U}_{\rightarrow}-Y_{r-}\right) d R_{r}^{-, *}+\sum_{0 \leq r<T_{a}}\left(U_{r}-Y_{r}\right) \Delta^{+} R_{r}^{-} \\
& \quad \leq \int_{0}^{T_{a}}\left(Y_{r-}^{2}-\overrightarrow{Y_{r}^{1}-U_{r}}\right) d K_{r}^{2, *}+\sum_{0 \leq r<T_{a}}\left(Y_{r}^{2}-\left(Y_{r}^{1}-U_{r}\right)\right) \Delta^{+} K_{r}^{2}=0 .
\end{aligned}
$$

Uniqueness of the solution follows from Proposition 4.3. Finally, by Proposition 4.3, $\bar{Y} \leq Y \leq \underline{Y}$, where $\underline{Y}$ (resp. $\bar{Y})$ is a solution to $\operatorname{RBSDE}^{T}(\xi, f+d V, L)\left(\right.$ resp. $\overline{\operatorname{RBSDE}}{ }^{T}(\xi, f+$ $d V, U)$ ). Therefore (6.1) follows from (H2) and Proposition 5.2.

\subsection{Nonlinear Dynkin games and stability results for RBSDEs}

Definition 6.2. Let $\tau \in \mathcal{T}$ and $H \in \mathcal{F}_{\tau}$. Write $H^{c}=\Omega \backslash H$. If $H^{c} \cap\{\tau=T\}=\emptyset$, then the pair $\rho=(\tau, H)$ is called a stopping system.

We denote by $\mathcal{S}$ the set of all stopping systems and for fixed stopping times $\sigma, \gamma \in \mathcal{T}$ we denote by $\mathcal{S}_{\sigma, \gamma}$ the set of stopping systems $\rho=(\tau, H)$ such that $\sigma \leq \tau \leq \gamma$. We put $\mathcal{S}_{\sigma}:=\mathcal{S}_{\sigma, T}$. Note that any stopping time $\tau \in \mathcal{T}$ can be identified with a stopping system $(\tau, \Omega)$. Therefore we may write $\mathcal{T} \subset \mathcal{S}$.

For a stopping system $\rho=(\tau, H)$ and for an optional process $X$, we set

$$
X_{\rho}^{u}=X_{\tau} \mathbf{1}_{H}+\overleftarrow{X}_{\tau} \mathbf{1}_{H^{c}}, \quad X_{\rho}^{l}=X_{\tau} \mathbf{1}_{H}+\underline{X}_{\tau} \mathbf{1}_{H^{c}} .
$$

Repeating step by step the proofs of [14, Lemma 4.15, Lemma 4.17] with using Propositions 3.4 and 4.3 we get the following result.

Theorem 6.3 (Dynkin games value process representation). Assume (H1)-(H4). Let 
$(Y, M, R)$ be a solution to $\operatorname{RBSDE}^{T}(\xi, f+d V, L, U)$. Then for every $\alpha \in \mathcal{T}$,

$$
\begin{aligned}
Y_{\alpha} & =\operatorname{essip}_{\rho=(\tau, H) \in \mathcal{S}_{\alpha}} \operatorname{essinf}_{\delta=(\sigma, G) \in \mathcal{S}_{\alpha}} \mathcal{E}_{\alpha, \tau \wedge \sigma}^{f}\left(\int_{\alpha}^{\tau \wedge \sigma} d V_{r}+L_{\rho}^{u} \mathbf{1}_{\{\tau \leq \sigma<T\}}+U_{\delta}^{l} \mathbf{1}_{\{\sigma<\tau\}}+\xi \mathbf{1}_{\{\tau=\sigma=T\}}\right) \\
& =\operatorname{essinf}_{\delta=(\sigma, G) \in \mathcal{S}_{\alpha}} \operatorname{essips}_{\rho=(\tau, H) \in \mathcal{S}_{\alpha}} \mathcal{E}_{\alpha, \tau \wedge \sigma}^{f}\left(\int_{\alpha}^{\tau \wedge \sigma} d V_{r}+L_{\rho}^{u} \mathbf{1}_{\{\tau \leq \sigma<T\}}+U_{\delta}^{l} \mathbf{1}_{\{\sigma<\tau\}}+\xi \mathbf{1}_{\{\tau=\sigma=T\}}\right) .
\end{aligned}
$$

Remark 6.4. The proof of (6.8) is much more simpler than the proof of the corresponding result for one barrier (Theorem 5.8). This is due to the fact that in (6.8) we can always indicate $\varepsilon$-optimal stopping systems regardless on the regularity of barriers $L, U$. These $\varepsilon$ optimal stopping systems $\rho_{\varepsilon}=\left(\tau_{\varepsilon}, H_{\varepsilon}\right), \delta_{\varepsilon}=\left(\sigma_{\varepsilon}, G_{\varepsilon}\right)$ are given by the following formulas (see $[14,(4.19)])$,

$$
\tau_{\varepsilon}=\inf \left\{t \geq \alpha ; Y_{t} \leq L_{t}+\varepsilon\right\} \wedge T, \quad \sigma_{\varepsilon}=\inf \left\{t \geq \alpha ; Y_{t} \geq U_{t}-\varepsilon\right\} \wedge T
$$

and

$$
H_{\varepsilon}=\left\{\omega \in \Omega ; Y_{\tau_{\varepsilon}(\omega)}(\omega) \leq L_{\tau_{\varepsilon}(\omega)}(\omega)+\varepsilon\right\}, \quad G_{\varepsilon}=\left\{\omega \in \Omega ; Y_{\sigma_{\varepsilon}(\omega)}(\omega) \geq U_{\sigma_{\varepsilon}(\omega)}(\omega)+\varepsilon\right\} .
$$

Note also that formulas of type (6.8) for linear RBSDEs (however without using the notion of RBSDEs) were proved in [2].

As a corollary to the above theorem we obtain a stability result for solutions to RBSDEs. Before stating it, we give some remarks about the process $\overleftarrow{X}$. Assume that $X$ is positive. From the definition it follows easily that $\overleftarrow{X}$ is the smallest progressively measurable process majorizing $X$ which is u.s.c. from the right. Let

$$
S_{\alpha}=\operatorname{esssup}_{\tau \geq \alpha} E\left(X_{\tau} \mid \mathcal{F}_{\alpha}\right), \quad \alpha \in \mathcal{T} \text {. }
$$

By [9, page 417], $S$ is the smallest supermartingale majorizing $X$, so $S$ is u.s.c. from the right and $X \leq S$. Thus $\overleftarrow{X} \leq S$. Therefore $\overleftarrow{X}$ is of class (D) and

$$
\|\overleftarrow{X}\|_{1} \leq\|S\|_{1}=\|X\|_{1}
$$

Corollary 6.5 (Stability of solutions to RBSDEs: case I). Assume that $\left(\xi_{i}, f_{i}, L^{i}, U^{i}\right), i=$ 1,2 , satisfy (H1)-(H4). Let $\left(Y^{i}, M^{i}, R^{i}\right)$ be a solution to $\operatorname{RBSDE}^{T}\left(\xi_{i}, f_{i}, L^{i}, U^{i}\right), i=1,2$. Then

$$
\begin{aligned}
\left\|Y^{1}-Y^{2}\right\|_{1} \leq & E\left|\xi_{1}-\xi_{2}\right|+2\left\|L^{1}-L^{2}\right\|_{1}+2\left\|U^{1}-U^{2}\right\|_{1} \\
& +\sup _{\rho, \delta \in \mathcal{S}} E \int_{0}^{\sigma \wedge \tau}\left|f_{1}-f_{2}\right|\left(r, \mathcal{E}_{r, \tau \wedge \sigma}^{f_{1}}\left(Z^{\rho, \delta}\right)\right) d r
\end{aligned}
$$

where

$$
Z^{\rho, \delta}=L_{\rho}^{u} \mathbf{1}_{\{\tau \leq \sigma<T\}}+U_{\delta}^{l} \mathbf{1}_{\{\sigma<\tau\}}+\xi \mathbf{1}_{\{\tau=\sigma=T\}} .
$$

Proof. By Theorem 6.3, $Y^{i}, i=1,2$, admits representation (6.8). Observe that

$$
\left|L_{\rho}^{1, u}-L_{\rho}^{2, u}\right| \leq\left|L_{\tau}^{1}-L_{\tau}^{2}\right|+\left.\overleftarrow{\mid L^{1}-L^{2}}\right|_{\tau}, \quad\left|U_{\delta}^{1, l}-U_{\delta}^{2, l}\right| \leq\left|U_{\sigma}^{1}-U_{\sigma}^{2}\right|+\left.\overleftarrow{\mid L^{1}-L^{2}}\right|_{\sigma}
$$

By this, (6.8) and Proposition 3.4,

$$
\begin{aligned}
E\left|Y_{\alpha}^{1}-Y_{\alpha}^{2}\right| \leq & \sup _{\tau, \sigma \in \mathcal{T}_{\alpha}} E\left(\left|\xi_{1}-\xi_{2}\right|+\left|L_{\tau}^{1}-L_{\tau}^{2}\right|+\overleftarrow{\left|L^{1}-L^{2}\right|_{\tau}}+\left|U_{\sigma}^{1}-U_{\sigma}^{2}\right|+\overleftarrow{\left.\left|L^{1}-L^{2}\right|_{\sigma}\right)}\right. \\
& +\sup _{\rho, \delta \in \mathcal{S}_{\alpha}} E \int_{\alpha}^{\sigma \wedge \tau}\left|f_{1}-f_{2}\right|\left(r, \mathcal{E}_{r, \tau \wedge \sigma}^{f_{1}}\left(Z^{\rho, \delta}\right)\right) d r
\end{aligned}
$$

Now, using (6.9), we get the desired result. 
Remark 6.6. Maintain the notation and the assumptions of Corollary 6.5. In case $f_{1}\left(\cdot, Y^{2}\right), f_{2}\left(\cdot, Y^{2}\right) \in L^{1}([0, T] \times \Omega ; P)$, we infer from this corollary that

$$
\left\|Y^{1}-Y^{2}\right\|_{1} \leq E\left|\xi_{1}-\xi_{2}\right|+2\left\|L^{1}-L^{2}\right\|_{1}+2\left\|U^{1}-U^{2}\right\|_{1}+E \int_{0}^{T}\left|f_{1}-f_{2}\right|\left(r, Y_{r}^{2}\right) d r .
$$

This is a consequence of the fact that $\left(Y^{2}, M^{2}, R^{2}\right)$ may be regarded as a solution to $\operatorname{RBSDE}^{T}\left(\xi, g+f^{1}, L, Y\right)$ with $g=f_{1}\left(\cdot, Y^{2}\right)-f_{2}\left(\cdot, Y^{2}\right)$. Thus, we get the analogue of [14, Corollary 5.2] and [15, Proposition 11.2].

Proposition 6.7 (Stability of solutions to RBSDEs: case II). Assume that $f, f_{n}, n \geq 1$, satisfy (H1)-(H4) and $f_{n} \nearrow f$ as $n \rightarrow \infty$. Let $\left\{L^{n}\right\}$ be a sequence of optional processes of class (D) on $[0, T]$ such that $L^{n} \nearrow L$.

(i) Let $(Y, M, K)$ (resp. $\left(Y^{n}, M^{n}, K^{n}\right)$ ) be a solution to $\underline{R B S D E}^{T}(\xi, f+d V, L)$ (resp. $\left.\underline{\operatorname{RBSDE}}^{T}\left(\xi, f+d V, L_{n}\right)\right)$. Then $Y^{n} \nearrow Y$.

(ii) Let $(Y, M, R)$ (resp. $\left(Y^{n}, M^{n}, R^{n}\right)$ ) be a solution of $\operatorname{RBSDE}^{T}(\xi, f+d V, L, U)$ (resp. $\left.\operatorname{RBSDE}^{T}\left(\xi, f_{n}+d V, L, U\right)\right)$ such that $Y$ (resp. $\left.Y^{n}\right)$ is of class (D). Then $\left\|Y-Y^{n}\right\|_{1} \rightarrow 0$ as $n \rightarrow \infty$.

Proof. (i) By Theorem 5.8 and Proposition 3.4(ii), for every $\alpha \in \mathcal{T}$,

$$
\begin{aligned}
Y_{\alpha}^{n} & =\operatorname{ess~sup}_{\tau \in \mathcal{T}_{\alpha}} \mathcal{E}_{\alpha, \tau}^{f}\left(\int_{\alpha}^{\tau} d V_{r}+L_{\tau}^{n} \mathbf{1}_{\tau<T}+\xi \mathbf{1}_{\tau=T}\right) \\
& \leq \operatorname{ess~sup}_{\tau \in \mathcal{T}_{\alpha}} \mathcal{E}_{\alpha, \tau}^{f}\left(\int_{\alpha}^{\tau} d V_{r}+L_{\tau} \mathbf{1}_{\tau<T}+\xi \mathbf{1}_{\tau=T}\right)=Y_{\alpha} .
\end{aligned}
$$

By Proposition 3.4(ii), $Y^{n} \leq Y^{n+1}, n \geq 1$. Set $X:=\sup _{n \geq 1} Y^{n}$. Then $X \leq Y$. For the opposite inequality first observe that for any $\alpha \in \mathcal{T}, \tau \in \mathcal{T}_{\alpha}$ and $n \geq 1$,

$$
X_{\alpha} \geq \underset{\tau \in \mathcal{T}_{\alpha}}{\operatorname{ess} \sup _{\alpha, \tau}} \mathcal{E}_{\alpha}^{f}\left(\int_{\alpha}^{\tau} d V_{r}+L_{\tau}^{n} \mathbf{1}_{\tau<T}+\xi \mathbf{1}_{\tau=T}\right) \geq \mathcal{E}_{\alpha, \tau}^{f}\left(\int_{\alpha}^{\tau} d V_{r}+L_{\tau}^{n} \mathbf{1}_{\tau<T}+\xi \mathbf{1}_{\tau=T}\right) .
$$

By Proposition 3.4(iii), $\mathcal{E}_{\alpha, \tau}^{f}\left(\int_{\alpha}^{\tau} d V_{r}+L_{\tau}^{n} \mathbf{1}_{\tau<T}+\xi \mathbf{1}_{\tau=T}\right) \rightarrow \mathcal{E}_{\alpha, \tau}^{f}\left(\int_{\alpha}^{\tau} d V_{r}+L_{\tau} \mathbf{1}_{\tau<T}+\xi \mathbf{1}_{\tau=T}\right)$ a.s. Hence

$$
X_{\alpha} \geq \mathcal{E}_{\alpha, \tau}^{f}\left(\int_{\alpha}^{\tau} d V_{r}+L_{\tau} \mathbf{1}_{\tau<T}+\xi \mathbf{1}_{\tau=T}\right)
$$

for any $\alpha \in \mathcal{T}$ and $\tau \in \mathcal{T}_{\alpha}$. Thus

$$
X_{\alpha} \geq \underset{\tau \in \mathcal{T}_{\alpha}}{\operatorname{ess} \sup _{\alpha, \tau}} \mathcal{E}_{\alpha}^{f}\left(\int_{\alpha}^{\tau} d V_{r}+L_{\tau} \mathbf{1}_{\tau<T}+\xi \mathbf{1}_{\tau=T}\right)=Y_{\alpha}, \quad \alpha \in \mathcal{T} .
$$

(ii) For each $n \geq 1, f_{n} \leq f_{n+1}$, so by Proposition $4.3, Y^{n} \leq Y^{n+1}$. Set $\tilde{Y}:=\sup _{n \geq 1} Y^{n}$. By Proposition 4.3

$$
Y^{1} \leq Y^{n} \leq Y, \quad n \geq 1
$$

Thus, $\tilde{Y}$ is of class (D) and $\lim _{a \rightarrow \infty} \tilde{Y}_{T_{a}}=\xi$. We will show that $Y=\tilde{Y}$. By (H2),

$$
\operatorname{sgn}\left(Y_{r}-Y_{r}^{n}\right)\left(f\left(r, Y_{r}\right)-f_{n}\left(r, Y_{r}^{n}\right)\right) \leq\left|f\left(r, Y_{r}\right)-f_{n}\left(r, Y_{r}\right)\right|
$$

By the minimality conditions for $R$ and $R^{n}$ (cf. Definition 4.2(b)),

$$
\operatorname{sgn}\left(Y_{r-}-Y_{r-}^{n}\right) d\left(R_{r}^{1}-R_{r}^{2}\right)^{*} \leq \mathbf{1}_{\left\{Y_{r-}>Y_{r-}^{n}\right\}} d R^{*,+}+\mathbf{1}_{\left\{Y_{r-}>Y_{r-}^{n}\right\}} d R^{n,-, *}=0
$$

and

$$
\operatorname{sgn}\left(Y_{r}-Y_{r}^{n}\right) \Delta^{+}\left(R_{r}-R_{r}^{n}\right) \leq \mathbf{1}_{\left\{Y_{r}>Y_{r}^{n}\right\}} \Delta^{+} R_{r}^{+}+\mathbf{1}_{\left\{Y_{r}>Y_{r}^{n}\right\}} \Delta^{+} R_{r}^{n,-}=0 .
$$


By [25, Corollary A.5], for all $a \geq 0$ and stopping times $\sigma, \tau \in \mathcal{T}^{T_{a}}$ such that $\sigma \leq \tau$, we have

$$
\begin{aligned}
\left|Y_{\sigma}-Y_{\sigma}^{n}\right| \leq & \left|Y_{\tau}-Y_{\tau}^{n}\right|+\int_{\sigma}^{\tau} \operatorname{sgn}\left(Y_{r}-Y_{r}^{n}\right)\left(f\left(r, Y_{r}\right)-f_{n}\left(r, Y_{r}^{n}\right)\right) d r \\
& +\int_{\sigma}^{\tau} \operatorname{sgn}\left(Y_{r-}-Y_{r-}^{n}\right) d\left(R_{r}^{1}-R_{r}^{2}\right)^{*}+\sum_{\sigma \leq r<\tau} \operatorname{sgn}\left(Y_{r}-Y_{r}^{n}\right) \Delta^{+}\left(R_{r}^{1}-R_{r}^{2}\right) \\
& -\int_{\sigma}^{\tau} \operatorname{sgn}\left(Y_{r-}-Y_{r-}^{n}\right) d\left(M_{r}-M_{r}^{n}\right) .
\end{aligned}
$$

By the above inequality and (6.11)-(6.13),

$$
\begin{aligned}
\left|Y_{\sigma}-Y_{\sigma}^{n}\right| \leq & \left|Y_{\tau}-Y_{\tau}^{n}\right|+\int_{\sigma}^{\tau}\left|f\left(r, Y_{r}\right)-f_{n}\left(r, Y_{r}\right)\right| d r \\
& -\int_{\sigma}^{\tau} \operatorname{sgn}\left(Y_{r-}-Y_{r-}^{n}\right) d\left(M_{r}-M_{r}^{n}\right) .
\end{aligned}
$$

By (H2), (6.10) and the fact that $f_{n} \leq f_{n+1}$ and $f_{n} \leq f$ we have

$$
f_{1}\left(r, Y_{r}\right) \leq f_{n}\left(r, Y_{r}\right) \leq f\left(r, Y_{r}^{1}\right)
$$

Let

$$
\sigma_{k}=\inf \left\{t \geq 0: \int_{0}^{t}\left|f_{1}\left(r, Y_{r}\right)\right|+\left|f\left(r, Y_{r}^{1}\right)\right| d r \geq k\right\} \wedge T .
$$

Observe that by (H4), $\left\{\sigma_{k}\right\}$ is a chain on $[0, T]$. By the definition of $\left\{\sigma_{k}\right\}$, (H4) and (6.15),

$$
E \int_{0}^{\sigma_{k}}\left|f\left(r, Y_{r}\right)-f_{n}\left(r, Y_{r}\right)\right| d r \rightarrow 0
$$

as $n \rightarrow \infty$. Let $\left\{\gamma_{k}\right\}$ be a fundamental sequence on $\left[0, T_{a}\right]$ for the local martingale $\int_{0}^{*} \operatorname{sgn}\left(Y_{r-}-Y_{r-}^{n}\right) d\left(M_{r}-M_{r}^{n}\right)$. By (6.14) with $\tau$ replaced by $\tau_{k}=\sigma_{k} \wedge \gamma_{k}, \tau_{k} \geq \sigma$ we have

$$
\begin{aligned}
\left|Y_{\sigma}-Y_{\sigma}^{n}\right| \leq & \left|Y_{\tau_{k}}-Y_{\tau_{k}}^{n}\right|+\int_{\sigma}^{\tau_{k}}\left|f\left(r, Y_{r}\right)-f_{n}\left(r, Y_{r}\right)\right| d r \\
& -\int_{\sigma}^{\tau_{k}} \operatorname{sgn}\left(Y_{r-}-Y_{r-}^{n}\right) d\left(M_{r}-M_{r}^{n}\right) .
\end{aligned}
$$

Since $Y$ and $Y^{n}$ are of class (D) and (6.16) holds true, we get, by taking the expectation in (6.17), letting $n \rightarrow \infty$ and then $k \rightarrow \infty$, that $E\left|Y_{\sigma}-\tilde{Y}_{\sigma}\right| \leq E\left|Y_{T_{a}}-\tilde{Y}_{T_{a}}\right|$ for $a \geq 0$. Now, letting $a \rightarrow \infty$, and using the fact that $Y, \tilde{Y}$ are of class (D), we get $E\left|Y_{\sigma}-\tilde{Y}_{\sigma}\right|=0$. Therefore, by the Section Theorem (see, e.g., [8, Chapter IV, Theorem 86]), $\left|Y_{t}-\tilde{Y}_{t}\right|=0$, $t \in[0, T]$, so $Y=\tilde{Y}$.

\subsection{Existence for RBSDEs under Mokobodzki's condition}

Theorem 6.8 (The existence result for two barrier RBSDEs: the general case). Assume that $(\mathrm{H} 1)-(\mathrm{H} 4),(\mathrm{H} 6)$ are satisfied. Then there exists a unique solution $(Y, M, R)$ to $\operatorname{RBSDE}^{T}(\xi, f+d V, L, U)$ such that $Y$ is of class (D).

Proof. Let $X$ be the process appearing in condition (H6). Since $X$ is a special semimartingale, there exists an increasing sequence $\left\{\gamma_{k}\right\} \subset \mathcal{T}$ such that $X$ is a difference of supermartingales of class (D) on $\left[0, \gamma_{k}\right]$ for every $k \geq 1$. Let $\varrho$ be a strcitly positive Borel measurable function on $\mathbb{R}^{+}$such that $\int_{0}^{\infty} \varrho(t) d t<\infty$. Set $\varrho_{n}(t)=\frac{n^{2} \varrho(t)}{1+n \varrho(t)}$. We let

$$
f_{n, m}(t, y)=\max \left\{\min \left\{f(t, y), \varrho_{n}(t)\right\},-\varrho_{m}(t)\right\} .
$$


Observe that $f_{n, m}$ is non-decreasing with respect to $n$ and non-increasing with respect to $m$. Moreover, $f_{n, m}(t, y) \nearrow f_{m}(t, y):=\max \left\{f(t, y),-\varrho_{m}(t)\right\}$ as $n \rightarrow \infty$ and $f_{m}(t, y) \searrow$ $f(t, y)$ as $m \rightarrow \infty$. Let $\hat{L}, \hat{U}$ be regulated processes defined by

$$
\hat{L}_{\alpha}=\underset{\tau \in \mathcal{T}_{\alpha}}{\operatorname{essinf}} E\left(L_{\tau} \mid \mathcal{F}_{\alpha}\right), \quad \hat{U}_{\alpha}=\operatorname{essiup}_{\tau \in \mathcal{T}_{\alpha}} E\left(U_{\tau} \mid \mathcal{F}_{\alpha}\right), \quad \alpha \in \mathcal{T}
$$

By Proposition 5.2, $-\hat{L}, \hat{U}$ are supermartingales of class (D) on $[0, T]$. Clearly, $\hat{L} \leq L \leq$ $U \leq \hat{U}$. Since $\hat{L}, \hat{U}$ are of class (D) on $[0, T]$, we get, by using (H4), that there exists a chain $\left\{\tau_{k}\right\}$ on $[0, T]$ such that

$$
E \int_{0}^{\tau_{k}}\left|f\left(r, \hat{L}_{r}\right)\right| d r+E \int_{0}^{\tau_{k}}\left|f\left(r, \hat{U}_{r}\right)\right| d r \leq k, \quad k \geq 1 .
$$

By replacing $\gamma_{k}$ by $\gamma_{k} \wedge \tau_{k}$ we may assume that $\gamma_{k} \leq \tau_{k}$. We define

$$
L_{t}^{n}=L_{t} \mathbf{1}_{\left\{t \leq \gamma_{n}\right\}}+\hat{L}_{t} \mathbf{1}_{\left\{t>\gamma_{n}\right\}}, \quad U_{t}^{n}=U_{t} \mathbf{1}_{\left\{t \leq \gamma_{n}\right\}}+\hat{U}_{t} \mathbf{1}_{\left\{t>\gamma_{n}\right\}} .
$$

Observe that

$$
\hat{L} \leq L^{n} \leq L^{n+1} \leq L \leq U \leq U^{n+1} \leq U^{n} \leq \hat{U}, \quad n \geq 1 .
$$

Moreover, $L^{n} \nearrow L$ and $U^{n} \searrow U$. Finally, we set

$$
X_{t}^{n, m}=X_{t} \mathbf{1}_{\left\{t \leq \gamma_{n} \wedge \gamma_{m}\right\}}+\hat{L}_{t} \mathbf{1}_{\left\{t>\gamma_{n} \geq \gamma_{m}\right\}}+\hat{U}_{t} \mathbf{1}_{\left\{t>\gamma_{m}>\gamma_{n}\right\}}
$$

Observe that $L^{n} \leq X^{n, m} \leq U^{m}$ and that the process $X^{n, m}$ is a difference of supermartingales of class (D) on $[0, T]$. Therefore, by the form of $f_{n, m},\left(\mathrm{H} 6^{*}\right)$ holds with $L^{n}, U^{m}$, $X^{n, m}$ and $f_{n, m}$. By Theorem 6.1, there exists a unique solution $\left(Y^{n, m}, M^{n, m}, R^{n, m}\right)$ of $\operatorname{RBSDE}^{T}\left(\xi, f_{n, m}, L^{n}, U^{m}\right)$ such that $Y^{n, m}$ is of class (D). By Proposition 4.3, $\left\{Y^{n, m}\right\}$ is non-decreasing with respect to $n$ and non-increasing with respect to $m$. Set

$$
Y^{m}=\sup _{n \geq 1} Y^{n, m}, \quad Y=\inf _{m \geq 1} Y^{m}
$$

Since $\hat{L} \leq Y^{n, m} \leq \hat{U}, n, m \geq 1$ and $L, U$ are of class (D), by the diagonal method we can find a subsequence $\left(m_{n}\right)$ of $(m)$ such that $\lim _{n \rightarrow \infty} E\left|Y_{\gamma_{k}}^{n, m_{n}}-Y_{\gamma_{k}}\right|=0$ for every $k \geq 1$. Let $k \leq n \wedge m$. By Theorem 6.3, for any $\alpha \in \mathcal{T}^{\gamma_{k}}$,

$$
\begin{aligned}
Y_{\alpha}^{n, m}=\operatorname{ess~sup}_{\rho=(\tau, H) \in \mathcal{S}_{\alpha, \gamma_{k}}} \operatorname{ess}_{\delta=(\sigma, G) \in \mathcal{S}_{\alpha, \gamma_{k}}} \mathcal{E}_{\alpha, \tau \wedge \sigma}^{f_{n, m}}\left(\int_{\alpha}^{\tau \wedge \sigma} d V_{r}\right. \\
\\
\left.\quad+L_{\rho}^{u} \mathbf{1}_{\left\{\tau \leq \sigma<\gamma_{k}\right\}}+U_{\delta}^{l} \mathbf{1}_{\{\sigma<\tau\}}+Y_{\gamma_{k}}^{n, m} \mathbf{1}_{\left\{\tau=\sigma=\gamma_{k}\right\}}\right) .
\end{aligned}
$$

Set, for every $\alpha \in \mathcal{T}^{\gamma_{k}}$,

$$
\begin{aligned}
Y^{(k)}(\alpha)= & \operatorname{essipp}_{\rho=(\tau, H) \in \mathcal{S}_{\alpha, \gamma_{k}}} \operatorname{essinf}_{\delta=(\sigma, G) \in \mathcal{S}_{\alpha, \gamma_{k}}} \mathcal{E}_{\alpha, \tau \wedge \sigma}^{f}\left(\int_{\alpha}^{\tau \wedge \sigma} d V_{r}\right. \\
& \left.+L_{\rho}^{u} \mathbf{1}_{\left\{\tau \leq \sigma<\gamma_{k}\right\}}+U_{\delta}^{l} \mathbf{1}_{\{\sigma<\tau\}}+Y_{\gamma_{k}} \mathbf{1}_{\left\{\tau=\sigma=\gamma_{k}\right\}}\right) .
\end{aligned}
$$

By Theorem 6.1 and Theorem 6.3, there exists a solution $\left(\tilde{Y}^{k}, \tilde{M}^{k}, \tilde{R}^{k}\right)$ to the problem $\operatorname{RBSDE}^{\gamma_{k}}\left(Y_{\gamma_{k}}, f, L, U\right)$ such that $Y^{(k)}(\alpha)=\tilde{Y}_{\alpha}, \alpha \in \mathcal{T}^{\gamma_{k}}$. By Remark 6.6,

$$
E\left|Y_{\alpha}^{n, m_{n}}-\tilde{Y}_{\alpha}^{k}\right| \leq E\left|Y_{\gamma_{k}}^{n, m_{n}}-Y_{\gamma_{k}}\right|+E \int_{\alpha}^{\tau \wedge \sigma}\left|f-f_{n, m_{n}}\right|\left(r, Y_{r}^{n, m_{n}}\right) d r
$$


By (6.18), (6.19), (H2) and the Lebesgue dominated convergence theorem,

$$
\begin{aligned}
& E \int_{0}^{\gamma_{k}}\left|f-f_{n, m_{n}}\right|\left(r, Y_{r}^{n, m_{n}}\right) d r \\
& \leq 2 E \int_{0}^{\tau_{k}} \mathbf{1}_{\left\{\left|f\left(r, \hat{L}_{r}\right)\right|+\left|f\left(r, \hat{U}_{r}\right)\right| \geq \varrho_{n}(r) \wedge \varrho_{m_{n}}(r)\right\}}\left(\left|f\left(r, \hat{L}_{r}\right)\right|+\left|f\left(r, \hat{U}_{r}\right)\right|\right) d r \rightarrow 0
\end{aligned}
$$

as $n \rightarrow \infty$. Thus, for any $k \geq 1, Y$ is the first component of a solution to the problem $\operatorname{RBSDE}^{\gamma_{k}}\left(Y_{\gamma_{k}}, f, L, U\right)$. What is left is to show that $Y_{T_{a}} \rightarrow \infty$ as $a \rightarrow \infty$. For this observe that by Proposition 4.3,

$$
\bar{Y}^{n, m} \leq Y^{n, m} \leq \underline{Y}^{n, m}, \quad n, m \geq 1
$$

where $\left(\underline{Y}^{n, m}, \underline{M}^{n, m}, \underline{K}^{n, m}\right)$ is a solution to $\underline{\operatorname{RBSDE}}^{T}\left(\xi, f_{m}, L^{n}\right)$ and $\left(\bar{Y}^{n, m}, \bar{M}^{n, m}, \bar{K}^{n, m}\right)$ is a solution to $\overline{\overline{\mathrm{R}}} \mathrm{BSDE}^{T}\left(\xi, f^{n}, U^{m}\right)$, where $f^{n}(t, y):=\min \left\{f(t, y), \varrho_{n}(t)\right\}$. By Proposition 6.7(ii), $\bar{Y}^{n, m} \nearrow \bar{Y}^{m}$, and by Proposition 6.7(i), $\underline{Y}^{n, m} \nearrow \underline{Y}^{m}$, where $\left(\bar{Y}^{m}, \bar{M}^{m}, \bar{K}^{m}\right)$ is a solution to $\overline{\operatorname{RBSDE}}{ }^{T}\left(\xi, f, U^{m}\right)$, and $\left(\underline{Y}^{m}, \underline{M}^{m}, \underline{K}^{m}\right)$ is a solution to $\underline{\operatorname{RBSDE}}^{T}\left(\xi, f_{m}, L\right)$. Therefore $\bar{Y}^{m} \leq Y^{m} \leq \underline{Y}^{m}$. Letting $m \rightarrow \infty$ and using once again Proposition 6.7 yields

$$
\bar{Y} \leq Y \leq \underline{Y}
$$

where $(\bar{Y}, \bar{M}, \bar{K})$ is a solution to $\overline{\operatorname{RBSDE}}{ }^{T}(\xi, f, U)$, and $(\underline{Y}, \underline{M}, \underline{K})$ is a solution to the problem $\underline{\operatorname{RBSDE}}^{T}(\xi, f, L)$. From this we get the desired result.

Theorem 6.9 (Existence for RBSDEs with non-integrable driver). Assume that (H2)-(H4), (H6) are satisfied. Furthermore, assume that $|V|_{T}<\infty$ a.s. Then there exists a unique solution $(Y, M, R)$ to $\operatorname{RBSDE}^{T}(\xi, f+d V, L, U)$ such that $Y$ is of class (D).

Proof. By (H4) and the assumption that $|V|_{T}<\infty$ a.s., there exists a chain $\left\{\delta_{k}\right\}$ on $[0, T]$ such that

$$
E \int_{0}^{\delta_{k}}|f(r, 0)| d r+E \int_{0}^{\delta_{k}} d|V|_{r}<\infty, \quad k \geq 1 .
$$

Let $f_{n, m}$ be as in the proof of Theorem 6.8. Set $V_{t}^{n, m}=V_{t}^{+} \wedge n-V_{t}^{-} \wedge m$. By Theorem 6.8 there exists a solution $\left(Y^{n, m}, M^{n, m}, R^{n, m}\right)$ of $\operatorname{RBSDE}^{T}\left(\xi, f_{n, m}+d V_{n, m}, L, U\right)$ such that $Y^{n, m}$ is of class (D). By Proposition 4.3, $\left\{Y^{n, m}\right\}$ is non-decreasing with respect to $n$ and non-increasing with respect to $m$. Now, repeating step by step the reasoning (6.20)-(6.21) of the proof of Theorem 6.8 with $\gamma_{k}$ replaced by $\delta_{k}$, we get that $Y$ defined by (6.20) (but with $Y^{n, m}$ defined as above) is the first component of the solution to $\operatorname{RBSDE}^{\delta_{k}}\left(Y_{\delta_{k}}, f, L, U\right)$ for any $k \geq 1$. It remains to show that $Y_{T_{a}} \rightarrow \xi$ as $a \rightarrow \infty$. We can not argue as in the proof of Theorem 6.8, because in general, under the assumptions of the present theorem, there are no solutions to $\overline{\operatorname{RBSDE}}{ }^{T}(\xi, f, U)$ and $\operatorname{RBSDE}^{T}(\xi, f, L)$. To get the desired convergence, we shall utilize the fact that $\left\{\delta_{k}\right\}$ is a chain. By the definition of a solution to $\operatorname{RBSDE}^{T}\left(Y_{\delta_{k}}, f, L, U\right), Y_{\delta_{k} \wedge a} \rightarrow Y_{\delta_{k}}$ as $a \rightarrow \infty$ for every $k \geq 1$. Since $\left\{\delta_{k}\right\}$ is a chain, for any $\omega \in \Omega$ there exists $k_{\omega} \geq 1$ such that $\delta_{k}(\omega)=T(\omega), k \geq k_{\omega}$. Therefore $Y_{T_{a}} \rightarrow \xi$ as $a \rightarrow \infty$ since by (6.20), $Y_{T}=\xi$.

\section{References}

[1] Akdim, K. and Ouknine, Y.: Infinite horizon reflected backward SDEs with jumps and RCLL obstacle. Stoch. Anal. Appl. 24, (2006), 1239-1261. MR-2273779

[2] Alario-Nazaret, M., Lepeltier, J. P. and Marchal, B.: Dynkin games (Bad Honnef Workshop on stochastic processes). Lect. Notes Control Inf. Sci. 43, (1982), 23-32. Springer-Verlag, Berlin. MR-0814103 
[3] Baadi, B. and Ouknine, Y.: Reflected BSDEs when the obstacle is not right-continuous in a general filtration. ALEA Lat. Am. J. Probab. Math. Stat. 14, (2017), 201-218. MR-3655127

[4] Baadi, B. and Ouknine, Y.: Reflected BSDEs with optional barrier in a general filtration. Afr. Mat. 29, (2018), 1049-1064. MR-3869547

[5] Bayraktar, E. and Yao, S.: Doubly reflected BSDEs with integrable parameters and related Dynkin games. Stochastic Process. Appl. 125, (2015), 4489-4542. MR-3406594

[6] Bismut, J. M.: Sur un problème de Dynkin. Z. Wahrscheinlichkeitstheorie und Verw. Gebiete 39, (1977), 31-53. MR-0444218

[7] Cvitanic J. and Karatzas, I.: Backward Stochastic Differential Equations with Reflection and Dynkin Games. Ann. Probab. 24, (1996), 2024-2056. MR-1415239

[8] Dellacherie, C. and Meyer, P. A.: Probabilities and Potential. North-Holland Mathematics Studies, 29. North-Holland, Amsterdam-New York, 1978. viii+189 pp. MR-0521810

[9] Dellacherie, C. and Meyer, P.A.: Probabilities and potential. B. Theory of martingales. Translated from the French by J. P. Wilson. North-Holland Mathematics Studies, 72. North-Holland, Amsterdam, 1982. xvii+463 pp. MR-0745449

[10] Dudley, R.M. and Norvaiša, R.: Concrete functional calculus. Springer Monographs in Mathematics. Springer, New York, 2011. xii+671 pp. MR-2732563

[11] El Karoui, N.: Les aspects probabilites du controle stochastique. Lecture Notes in Math. 876, (1981), 73-238. MR-0637471

[12] El Karoui, N., Kapoudjian, C., Pardoux, E., Peng, S. and Quenez, M. C.: Reflected solutions of backward SDEs, and related obstacle problems for PDE's. Ann. Probab. 25, (1997), 702-737. MR-1434123

[13] Grigorova, M., Imkeller, P., Offen, E., Ouknine, Y. and Quenez, M. C.: Reflected BSDEs when the obstacle is not right-continuous and optimal stopping. Ann. Appl. Probab. 27, (2017), 3153-3188. MR-3719955

[14] Grigorova, M., Imkeller, P., Ouknine, Y. and Quenez, M. C.: Doubly Reflected BSDEs and $\mathcal{E}^{f}$-Dynkin games: beyond the right-continuous case. Electron. J. Probab. 23, (2018), Paper No. 122, 38 pp. MR-3896859

[15] Grigorova, M., Imkeller, P., Ouknine, Y. and Quenez, M. C.: Optimal stopping with $f-$ expectations: the irregular case. Stochastic Process. Appl. 130, (2020), 1258-1288. MR4058273

[16] Hamadène, S. and Hassani, M.: BSDEs with two reflecting barriers driven by a Brownian and a Poisson noise and related Dynkin game. Electron. J. Probab. 11, (2006), 121-145. MR-2217812

[17] Hamadène, S.: Reflected BSDEs with discontinuous barrier and applications. Stochastics Stochastics Rep. 74, (2002), 571-596. MR-1943580

[18] Hamadène, S. and Ouknine, Y.: Reflected backward stochastic differential equation with jumps and random obstacle. Electron. J. Probab. 8, (2003), no. 2, 20 pp. MR-1961164

[19] Hamadène, S. and Ouknine, Y.: Reflected backward SDEs with general jumps. Theory Probab. Appl. 60, (2016), 263-280. MR-3568776

[20] Hamadène, S. and Wang, H.: BSDEs with two RCLL reflecting obstacles driven by Brownian motion and Poisson measure and related mixed zero-sum game. Stochastic Process. Appl. 119, (2009), 2881-2912. MR-2554032

[21] Hamadène, S. and Popier, A.: $L^{p}$-solutions for reflected backward stochastic differential equations. Stoch. Dyn. 12, (2012), 1150016, 35 pp. MR-2902162

[22] Klimsiak, T.: BSDEs with monotone generator and two irregular reflecting barriers. Bull. Sci. Math. 137, (2013), 268-321. MR-3043077

[23] Klimsiak, T.: Reflected BSDEs on filtered probability spaces. Stochastic Process. Appl. 125, (2015), 4204-4241. MR-3385601

[24] Klimsiak, T.: Systems of quasi-variational inequalities related to the switching problem. Stochastic Process. Appl. 129, (2019), 1259-1286. MR-3926555

[25] Klimsiak, T., Rzymowski, M. and Słomiński, L.: Reflected BSDEs with regulated trajectories. Stochastic Process. Appl. 129, (2019), 1153-1184. MR-3926552 
[26] Klimsiak, T., Rzymowski, M. and Słomiński, L.: Reflected backward stochastic differential equations with two optional barriers. Bull. Sci. Math. 158, (2020), 102820, 49 pp. MR4030244

[27] Klimsiak, T.: Non-semimartingale solutions of reflected BSDEs and applications to Dynkin games. Stochastic Process. Appl. 134, (2021), 208-239. MR-4206845

[28] Lepeltier, J.P., Matoussi, A. and Xu, M.: Reflected backward stochastic differential equations under monotonicity and general increasing growth conditions. Adv. in Appl. Probab. 37, (2005), 134-159. MR-2135157

[29] Lepeltier, J.P. and Xu, M.: Reflected BSDEs with two rcll barriers. ESAIM Probab. Stat. 11, (2007), 3-22. MR-2299643

[30] Marzougue, M. and El Otmani, M.: BSDEs with right upper-semicontinuous reflecting obstacle and stochastic Lipschitz coefficient. Random Oper. Stoch. Equ. 27, (2019), 27-41. MR-3916509

[31] Marzougue, M. and El Otmani, M.: Non-continuous double barrier reflected BSDEs with jumps under a stochastic Lipschitz coefficient. Commun. Stoch. Anal. 12, (2018), Article 1, 359-381. MR-3957705

[32] Mertens J.F.: Théorie des processus stochastiques généraux applications aux surmartingales. Z. Wahr. verw. Geb. 22, (1972), 45-68. MR-0346895

[33] Peng, S.: Backward SDE and related g-expectation. Backward stochastic differential equations (Paris, 1995-1996). Pitman Res. Notes Math. Ser. 364, (1997), 141-159. Longman, Harlow. MR-1752680

[34] Rozkosz, A. and Słomiński, L.: $L^{p}$ solutions of reflected BSDEs under monotonicity condition. Stochastic Process. Appl. 122, (2012), 3875-3900. MR-2971718

[35] Rozkosz, A. and Słomiński, L.: Stochastic representation of entropy solutions of semilinear elliptic obstacle problems with measure data. Electron. J. Probab. 17, (2012), no. 40, pp. 27. MR-2928723

[36] Ren, Y. and El Otmani, M.: Generalized reflected BSDEs driven by a Lévy process and an obstacle problem for PDIEs with a nonlinear Neumann boundary condition. J. Comput. Appl. Math. 233, (2010), 2027-2043. MR-2564037 